\title{
Reconstruction and Quantification of the Carotid Artery Bifurcation From 3-D Ultrasound Images
}

\author{
Dean C. Barratt*, Member, IEEE, Ben B. Ariff, Keith N. Humphries, Simon A. McG. Thom, and Alun D. Hughes
}

\begin{abstract}
Three-dimensional (3-D) ultrasound is a relatively new technique, which is well suited to imaging superficial blood vessels, and potentially provides a useful, noninvasive method for generating anatomically realistic 3-D models of the peripheral vasculature. Such models are essential for accurate simulation of blood flow using computational fluid dynamics (CFD), but may also be used to quantify atherosclerotic plaque more comprehensively than routine clinical methods. In this paper, we present a spline-based method for reconstructing the normal and diseased carotid artery bifurcation from images acquired using a freehand 3-D ultrasound system. The vessel wall (intima-media interface) and lumen surfaces are represented by a geometric model defined using smoothing splines. Using this coupled wall-lumen model, we demonstrate how plaque may be analyzed automatically to provide a comprehensive set of quantitative measures of size and shape, including established clinical measures, such as degree of (diameter) stenosis. The geometric accuracy of 3-D ultrasound reconstruction is assessed using pulsatile phantoms of the carotid bifurcation, and we conclude by demonstrating the in vivo application of the algorithms outlined to 3-D ultrasound scans from a series of patient carotid arteries.
\end{abstract}

Index Terms-3-D ultrasound, carotid artery, plaque, quantification, reconstruction, stenosis.

\section{INTRODUCTION}

D EVELOPMENTS in three-dimensional (3-D) vascular imaging now make it possible to obtain anatomically accurate 3-D images of blood vessels. Magnetic resonance angiography (MRA) and X-ray computerized tomography (CT) angiography are becoming widely used for this purpose, but 3-D ultrasound has also been recognized as a potential alternative, particularly for the peripheral vessels [1]-[4]. In this paper, we focus on the generation and quantification of geometrically accurate models of the human carotid bifurcation using 3-D ultrasound. Such models are an essential prerequisite for accurate simulation of blood flow using computational fluid

Manuscript received November 14, 2003; revised January 19, 2004. This work was carried out as part of a clinical trial supported by Astra-Zeneca Plc. The Associate Editor responsible for coordinating the review of this paper and recommending its publication was W. J. Niessen. Asterisk indicates corresponding author.

*D. C. Barratt was with the Department of Clinical Pharmacology \& Therapeutics, National Heart \& Lung Institute, Imperial College London, U.K. He is now with the Computational Imaging Science Group, Division of Imaging Sciences, Floor 5, Thomas Guy House, Guy's Hospital, London, SE1 9RT U.K. (e-mail: dean.barratt@ kcl.ac.uk).

B. B. Ariff, S. A. M. Thom, and A. D. Hughes are with the Department of Clinical Pharmacology \& Therapeutics, National Heart \& Lung Institute, Imperial College London at St. Mary's Hospital, Paddington, London W2 1NY, U.K.

K. N. Humphries is with the Radiological Sciences Unit, Department of Imaging, Imperial College London at the Hammersmith Hospital, London W12 0NN, U.K.

Digital Object Identifier 10.1109/TMI.2004.825601 dynamics (CFD), with the general aim of further understanding how fluid mechanical factors are implicated in the development of atherosclerosis [5]-[8]. A second important clinical application is in the assessment of atherosclerotic plaque in the internal carotid artery (ICA), which is associated with risk of stroke and transient ischemic attack [9]-[12].

The conventional clinical measure of severity of atherosclerotic disease in the carotid arteries is degree of stenosis, defined as the percentage lumen diameter reduction relative to some reference vessel diameter. For instance, degree of stenosis as defined in the North American Symptomatic Carotid Endarterectomy Trial (NASCET) is [13]

$$
100 \times\left(1-\frac{d_{\mathrm{ICA}, \text { min }}}{d_{\mathrm{ICA}, \text { distal }}}\right)
$$

where $d_{\mathrm{ICA}, \min }$ is the minimum lumen diameter in the ICA (i.e., at the site of maximal stenosis) and $d_{\mathrm{ICA} \text {,distal }}$ is the lumen diameter in a distal diease-free portion of the ICA. Based on evidence from NASCET and the European Carotid Surgery Trial (ECST), carotid endarterectomy - the standard surgical intervention for carotid disease-significantly reduces the risk of stroke in recently symptomatic patients with a degree of stenosis above 70\% [14]-[16]. ${ }^{1}$ Although X-ray angiography is still widely considered to be the Gold Standard for determining degree of stenosis in the carotid arteries, two-dimensional (2-D) Duplex ultrasound imaging is a well-established alternative [10], [18], [19]. Ultrasound has the advantage that it is noninvasive and does not involve the use of ionizing radiation. It is, therefore, ideally suited to serial investigations. It is also relatively inexpensive and images are acquired in real-time. In practice, ICA stenosis is commonly estimated from blood velocity measurements made using Doppler ultrasound. Although this method has proven effective in identifying stenoses above the threshold for carotid endarterectomy, it is widely considered to be unsuitable for accurate quantification of disease severity over a wide range of degrees of stenosis [20]-[28]. In particular, it is not useful for quantifying low to moderate degrees of stenosis where there is no appreciable elevation in blood velocity, and is still used in many centers primarily as a screening tool to select patients for angiography.

Unlike alternative angiographic techniques-specifically, MRA and X-ray and CT angiography-modern ultrasound scanners have the ability to image blood flow and the soft tissue of the vessel wall simultaneously. When artifacts such as

\footnotetext{
${ }^{1}$ There is a disparity between the reported criteria for carotid endarterectomy recommended by the NASCET and ECST trials. However, recent reanalysis has shown that the results of the two trials are largely consistent [16], [17].
} 
acoustic shadowing do not obscure visualization of plaque, it is possible to measure plaque and vessel dimensions directly, rather than indirectly from localized narrowing of the vessel lumen as is the case with angiographic methods and Doppler ultrasound. Although this results in a definition of degree of stenosis that differs from that used in the NASCET or ECST trials, this method avoids inherent underestimation of plaque severity, which is one limitation to angiographic imaging, modality-specific artifacts notwithstanding. Underestimation of the amount of plaque is especially likely when the disease is diffuse or where significant remodeling of the vessel wall has taken place [29]-[32]. Direct measurement from B-mode images overcomes this limitation, and is valuable for quantification of low to moderate stenoses, which can be difficult to identify using other techniques.

Although Doppler ultrasound has largely superceded direct measurement in the quantification of carotid plaque in routine clinical practice, recent advances in ultrasound imaging have resulted in considerable improvements in image resolution and quality. This, combined with the well-documented limitations of Doppler ultrasound and developments in 3-D ultrasound imaging [33]-[41], provides an impetus for revisiting methods for quantifying atherosclerotic plaque directly from ultrasound images. This approach has already been investigated using conventional 2-D B-mode imaging [42], [43], but 3-D ultrasound has the potential to provide more accurate and comprehensive quantification of plaque.

To date, a number of researchers have investigated the use of 3-D ultrasound to quantify carotid plaque [44]-[56]. However, the majority of these studies have concentrated on measuring plaque dimensions and volume without considering the geometric relationship between plaque and the vessel wall. Knowledge of this relationship is important because it allows the true severity of disease to assessed. One exception is Yao and co-workers who also determined plaque length and the degree of stenosis from parallel cross-sectional slices [56]. A recent study by Gill et al. reported on the application of a two-step dynamic balloon method for modeling the lumen of the carotid bifurcation from 3-D ultrasound images [57]. Although the geometric accuracy and variability of the technique were found to be good compared with results based on manual segmentation, the algorithm was found to be sensitive to image speckle and model starting parameters. As a result, the surface mesh sometimes became self-intersecting if the model parameters were not chosen appropriately. Moreover, no attempt was made to quantify plaque from the model in this study.

Kovalski et al. developed an algorithm for 3-D reconstruction of a geometric model of the lumen and wall of coronary arteries from intravascular ultrasound images [58]. Although they suggest that the algorithm may provide an accurate clinical tool for quantitative assessment of the plaque and lumen, this was not explored.

The principal contribution of the work presented in the present paper is the development of methods for quantifying 3-D geometric surface models of normal and diseased carotid bifurcations reconstructed from noninvasive 3-D ultrasound images. A novel aspect of our approach is the coupled reconstruction of both the lumen and wall surfaces. A spline-based surface representation was chosen so that a degree of smoothing could be included in the reconstruction. Although smoothing is generally undesirable for the lumen surface in order to preserve small-scale plaque surface features, the vessel wall can be reasonably assumed to be smooth. The spline-based surface representation provides a compact surface representation from which precise geometric measurements can be computed automatically to an arbitrary resolution. In particular, we show how the combined wall-lumen model is used to calculate degree of stenosis within a transverse plane at any location along the ICA. Quantification of plaque volume, volumetric stenosis, plaque length and plaque distribution are also demonstrated. Similar ideas have been applied in order to monitor the progression of atherosclerotic disease in lower extremity bypass grafts and the 3-D geometry of abdominal aortic aneurysms [59]-[61], but we are not aware of any previous work to comprehensively quantify plaque in the carotid bifurcation using noninvasive 3-D ultrasound.

\section{THREE-DIMENSIONAL ULTRASOUND ACQUISITION}

The freehand 3-D ultrasound system used in this study was described previously in [62] and [63]. The system employs a pulsed-dc electromagnetic tracking device (pcBIRD, Ascension Technologies Inc., VT) and interfaces with a commercial ultrasound scanner used for routine vascular investigations (HDI-5000, ATL-Philips Ltd., Bothell, WA). The sensor of the tracking device is mounted on the ultrasound scan probe (L12-5, 5-12 MHz broadband, linear-array transducer) to enable 2-D ultrasound images to be located in 3-D space. Digital image and positional data were captured simultaneously at regular intervals, synchronized to an analogue trigger pulse generated by a data acquisition board in the host PC. To reduce artifacts due to the pulsatile vessel motion, each image was captured following a fixed delay $(\sim 400 \mathrm{~ms})$ relative to the peak of the electrocardiogram (ECG) R-wave. This ensured that images were obtained at a consistent point during the diastolic phase of the cardiac cycle. The trigger delay could be 'tuned' for individual subjects so that maximal color filling occurred when power mode imaging was used.

During acquisition, digital ultrasound image data were stored on the scanner in a prescan-converted format. At the end of each 3-D ultrasound acquisition, image data were downloaded to the host PC using a direct network connection and proprietary software, called HDILab, supplied by the ultrasound manufacturer. A 3-D ultrasound scan involved slowly sweeping the scan-probe across the skin surface with the scan-probe orientated so that transverse images of the vessel were acquired. During a typical 3-D ultrasound sweep, 50-140 images were captured.

In this paper, both B- and power mode images were obtained with a fixed depth setting of $4 \mathrm{~cm}$ and a single focal zone placed at a depth of $2 \mathrm{~cm}$. Power mode images comprise a grayscale B-mode image with the power Doppler signal displayed as a colored overlay, as shown in Fig. 1(a). Moving the scan-probe slowly ensured that so-called flash artifacts were avoided, and, since prescan-converted image data were stored, it was possible to separate the B-mode and color components using the HDILab software [see Fig. 1(b)]. This was useful as it meant that the color overlay could be turned off so that power Doppler artifacts did not adversely affect the image analysis. 


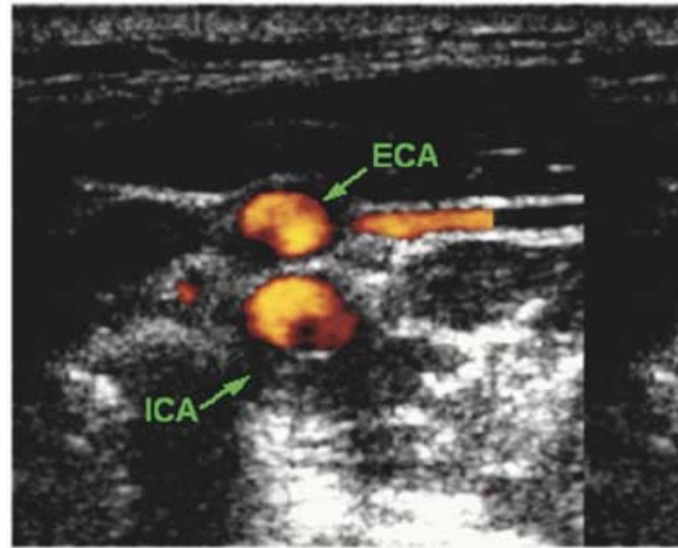

(a)

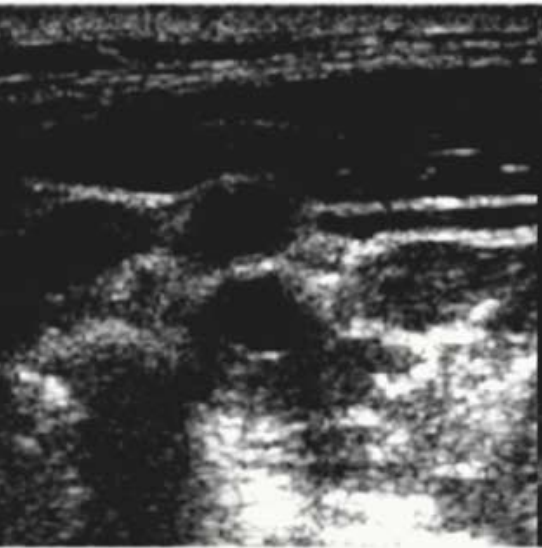

(b)

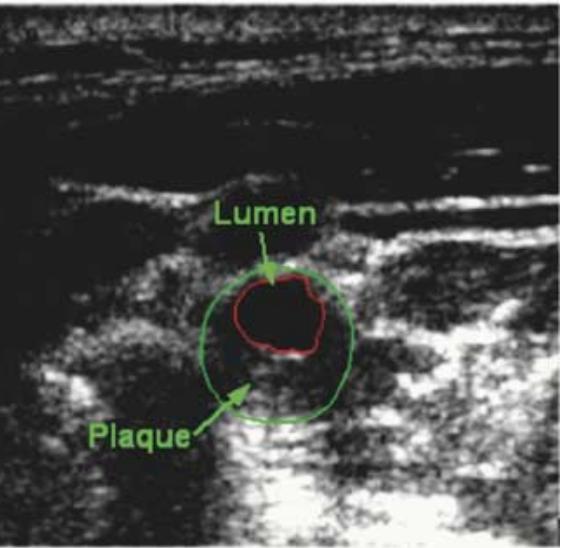

(c)

Fig. 1. (a) Example of a transverse power mode ultrasound image of the internal and external carotid arteries (labeled ICA and ECA, respectively). The orange region represents the color-coded power of the backscattered Doppler signal from flowing blood, superimposed onto the B-mode image. An atherosclerotic plaque is visible in the ICA. (b) The B-mode component of the image. (c) Segmented wall and lumen boundaries of the ICA (shown in green and red, respectively). The region between these boundaries is occupied by plaque.

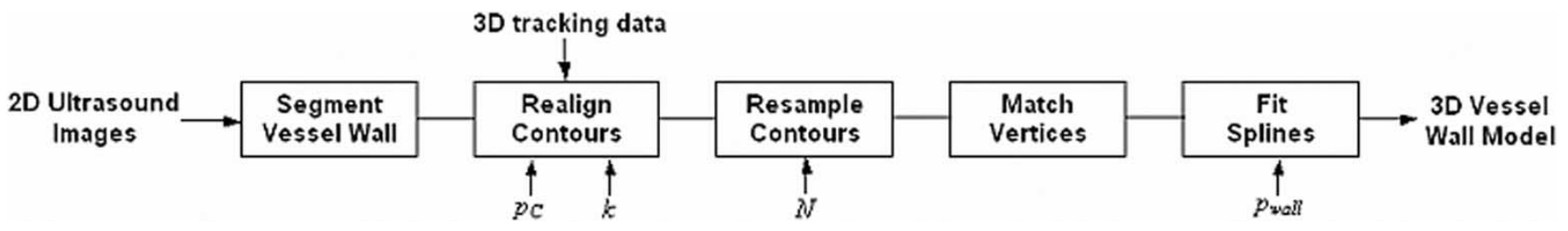

Fig. 2. Block diagram of the reconstruction of the wall of the carotid bifurcation. $p_{C}$ and $p_{\text {wall }}$ are smoothing parameters for the cubic smoothing spline defined in (2). $k$ is a positive integer, defined in (3), and $N$ sets the number of vertices in the polygonal approximation of cross-sectional vessel contours.

\section{RECONSTRUCTION OF THE VESSEL WALL}

There are numerous reports in the literature on the segmentation and 3-D reconstruction of blood vessels from medical images [8], [64]-[67]. The algorithm presented below is suitable for reconstructing vessel surfaces from cross-sectional contours extracted from a series of nonparallel 2-D images. Surfaces are represented using smoothing splines, which have the advantage that a variable degree of surface smoothing can be applied. However, any type of curve could be used in principle. A block diagram of the basic steps involved in the reconstruction of the vessel wall is shown in Fig. 2. Reconstruction of the lumen for the case of diseased arteries is described in Section IV.

\section{A. Preprocessing}

Ultrasound images acquired using the freehand 3-D ultrasound system were segmented manually with software written using Matlab version 6 (The Mathworks Inc., Natick, MA). The software provided an easy-to-use user interface for segmenting the vessel wall and lumen directly from the acquired ultrasound images. Overlapping ultrasound slices were removed prior to segmentation and, although the power Doppler component was found to be useful for locating the lumen, only the B-mode component of each image was used when delineating the wall and lumen boundaries in order to eliminate errors due to color artifact. For the purposes of this study, the vessel wall and lumen boundaries are defined as follows: the lumen is the boundary enclosing the interior region of the vessel through which blood flows. The lumen appears as a dark region in a B-mode image [see Fig. 1(b) and (c)]. The vessel wall is the boundary delineated by the intima-media interface. The intima-media interface is frequently visible in transverse ultrasound images, except in cases where artifacts-for example, an acoustic shadow cast by a dense or calcified plaque-may obscure visualization of this boundary.

On transverse images of the carotid arteries, the vessel wall was always defined by a closed contour, whereas plaque was delineated using either an open or closed contour, represented by a cubic spline. Since the radius of curvature of each carotid artery is usually very large compared with its diameter, and the rate of change of vessel wall diameter is slow, cross sections of the vessel wall could usually be approximated by an ellipse in all regions apart from the region immediately proximal to the flow divider. The vessel wall in the slice immediately proximal to the flow divider was defined as two overlapping ellipses. This led to a convenient representation for the bifurcation with the flow-divider defined implicitly by the intersection of the tubular models of the internal and external carotid arteries (see Section III-B below).

For simplicity, the vessel wall and lumen were assumed to be identical for young, healthy subjects, since the intima-media thickness (IMT) is very small. This is because the thin intimal layer tends only to be clearly visible in a transverse ultrasound image along a short segment of the inferior vessel wall, where the vessel is approximately perpendicular to the direction of the ultrasound beam. An improved estimate of the vessel lumen could be obtained by assuming a uniform IMT around the circumference of the vessel and subtracting this from the wall boundary. For many patients with atherosclerotic plaque, however, the lumen and wall boundaries are clearly distinguishable, with the region between corresponding to plaque [see Fig. 1(c)]. 
Before reconstruction, cross-sectional wall contours were shifted so that their centroids coincided with a smooth curve fitted to the original centroid positions [7]. This step was useful to remove small 3-D localization errors largely due to vessel movement caused by pulsatile blood flow or pressure applied by contact between the scan-probe and skin surface. Errors in contour positions may also occur if the scan-probe is moved rapidly due to the lack of synchrony between the image capture and position measurement caused by small delays inherent in the acquisition system. Although the sweep speed was limited to avoid this effect in the sweep direction, in practice, small but rapid translations of the scan-probe approximately perpendicular to the skin surface can occur involuntarily. These effects can sometimes result in a small number slices being significantly misaligned ( $>2 \mathrm{~mm}$ ), which may be considered as outliers. To compensate for such errors, a correction method was applied that is robust to outliers. The method described below was implemented using the Matlab Spline Toolbox version 3, and is only directly applicable to wall contours, where the assumption of a slowly changing centroid is valid. For the case of a stenosed vessel, the shift computed for each wall contour was also applied to the lumen contours as they are physically connected.

The wall contours for the internal, external and common carotid arteries were treated separately. A weighted, cubic smoothing spline, $f(s)$, was fitted to wall contour centroids for each artery by minimizing the expression [68]

$$
p_{C} \sum_{i} w_{i}\left(v_{i}-f\left(s_{i}\right)\right)^{2}+\left(1-p_{C}\right) \int_{0}^{L} f^{\prime \prime}(s)^{2} d s
$$

where $p_{C} \in[0,1]$ is a smoothing parameter; $w_{i}$ is a weight, which governs the relative influence of the $i$ th centroid; $v_{i}$ is the $x^{-}, y^{-}$, or $z$ component of the position vector that define the 3-D position of the $i$ th centroid; $s_{i}$ is the value of an arclength parameter $s(0 \leq s \leq L)$ for the $i$ th centroid; and $f^{\prime \prime}$ is the second derivative of $f$ with respect to $s$. The summation component of (2) represents a measure of error of fit to the input points (centroids in this case), whereas the integral term is a measure of smoothness. Therefore, setting $p_{C}=1$ results in a least-squares fit with no smoothing.

In order to ensure robustness in the presence of outliers, a weight, $w_{i}$, was calculated for each centroid in turn by removing it (equivalent to setting $w_{i}=0$ ), fitting a uniformly weighted cubic smoothing spline to the remaining centroids (i.e., $w_{j}=1$, $j \neq i$ ), and then computing the Euclidean distance $d_{i}$ between the removed centroid and the corresponding point on the fitted spline. The weight was then calculated using

$$
w_{i}=d_{i}^{-k}
$$

where $k$ is a positive scalar exponent. Empirically, setting $k$ to a value of 3 was found to work well for the data analyzed in this study.

After assigning values for all weights and fitting the weighted smoothing spline, each contour was translated within the sliceplane of the original acquired ultrasound image so that its centroid coincided with the intersection of the central axis curve and the image plane. This method assumes that misalignment

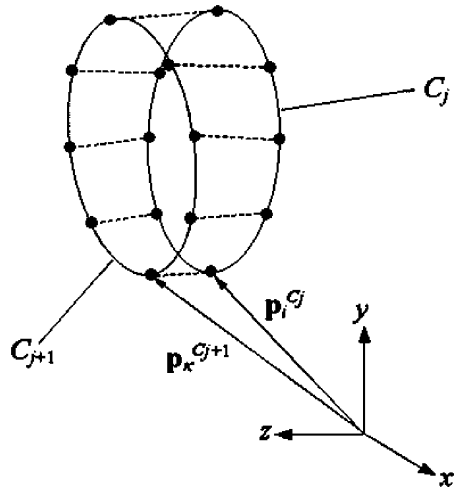

Fig. 3. The scheme used to match vertices on consecutive cross-sectional vessel contours, $C_{j}$ and $C_{j+1}$, where the total Euclidean distance between matched vertices is minimized.

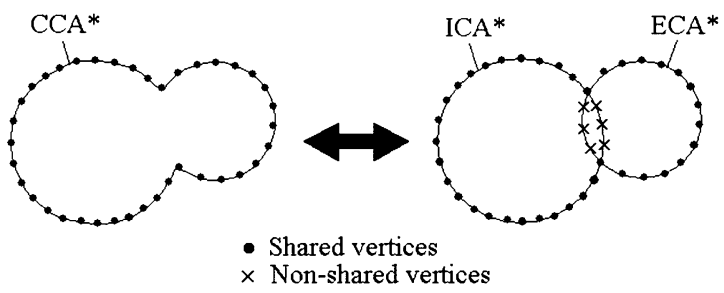

(a)

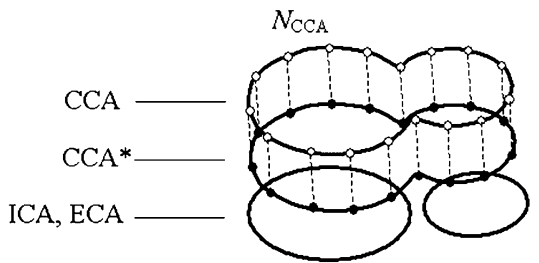

(b)

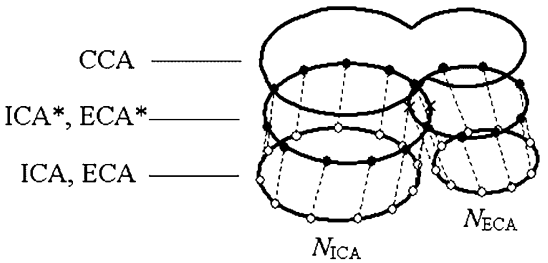

(c)

Fig. 4. Vertex matching scheme at the carotid bifurcation. (a) The common carotid contour, $\mathrm{CCA}^{*}$, immediately proximal to the bifurcation, is defined as the union of the two intersecting contours, ICA* and ECA*. (b) Vertex matching proximal to the bifurcation is achieved by matching the vertices of $\mathrm{CCA}^{*}$ to those of the proximal contour, CCA. (c) Vertex matching distal to the bifurcation is achieved by independently matching the vertices of ICA* and ECA* to distal ICA and ECA contours, respectively. In this way, the geometry of the bifurcation is approximated implicitly by the intersection of the internal and external carotid arteries (modeled as tubes).

occurs primarily within the original acquisition plane, as discussed above; it does not account for positional errors in the direction of the sweep or rotational errors, which are assumed to be negligible.

\section{B. Spline-Based Surface Reconstruction Method}

In the method proposed here, which is shown schematically in Fig. 5, the vessel wall surface is reconstructed by fitting longitudinally oriented splines to matched points from adjacent cross-sectional vessel contours. Cubic smoothing splines were chosen for this purpose because of their ability incorporate a 


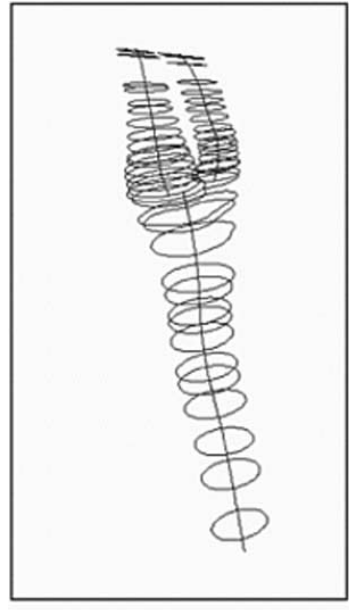

(a)

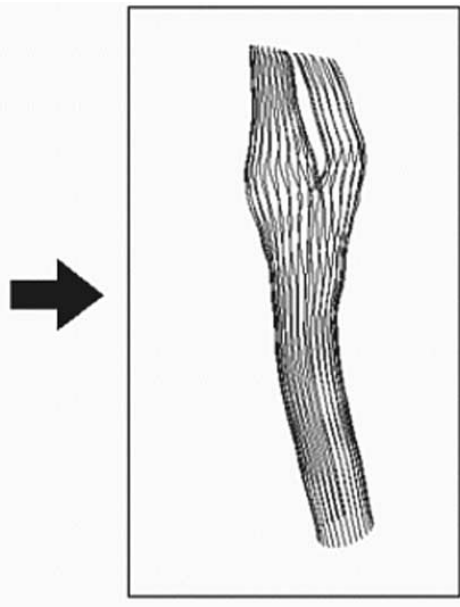

(b)

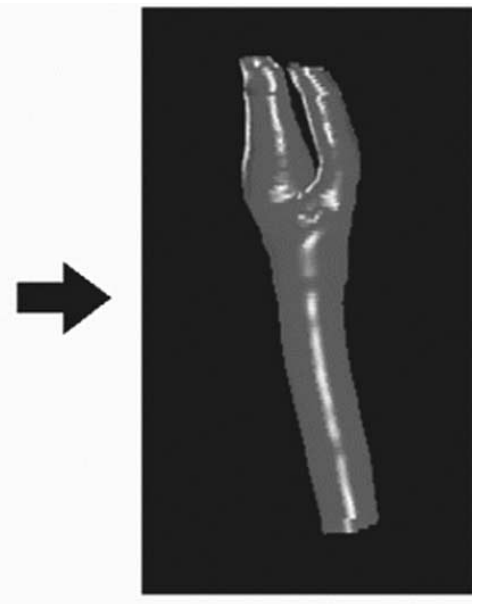

(c)

Fig. 5. Surface reconstruction of a patient's carotid artery bifurcation from a series of 2-D ultrasound images. (a) Cross-sectional wall contours located in 3-D following contour realignment. (b) Longitudinal smoothing splines fitted to matched vertices of adjacent contours. (c) Three-dimensional rendering of the reconstructed vessel wall surface.

variable degree of smoothing, determined by a smoothing parameter, $p_{\text {wall }}$. Initially, $N$ equally spaced points (typically, $N \geq$ 50) are computed around each wall contour. By analogy with polygonal representation of a closed contour, these points are referred to here as vertices. Vertices on consecutive contours are matched by finding the value of the positive integer, $\alpha$, that minimizes the Euclidean distance, $D$, defined by

$$
D=\sum_{i=0}^{N-1}\left|\mathbf{p}_{i}^{C_{j}}-\mathbf{p}_{(i+\alpha) \bmod N}^{C_{j+1}}\right|
$$

where $\mathbf{p}_{i}^{C_{j}}$ is the 3 -D position vector of the $i$ th vertex $(0 \leq$ $i \leq N-1$ ) of the $j$ th contour, $C_{j}$ (see Fig. 3). Longitudinal smoothing splines are then fitted to each set of matched vertices.

To handle the carotid bifurcation, the following adaptations were made to the basic method described above: with reference to Fig. 4(a), the contour of the common carotid artery (CCA*), immediately proximal to the flow divider is the union of the two overlapping internal and external carotid contours, defined explicitly during segmentation, and denoted by $\mathrm{ICA}^{*}$ and $\mathrm{ECA}^{*}$, respectively. Contour $\mathrm{CCA}^{*}$ shares all of its vertices with $\mathrm{ICA}^{*}$ and $\mathrm{ECA}^{*}$, and all common carotid contours proximal to the flow divider [CCA in Fig. 4(b)] are resampled so that they have the same number of vertices as CCA*. Distal to the flow divider, the number of vertices in $\mathrm{ICA}^{*}$ and $\mathrm{ECA}^{*}$ must correspond to the number of vertices in internal and external carotid artery contours (ICA and ECA, respectively), as indicated in Fig. 4(c). Nonshared vertices of ICA* and $\mathrm{ECA}^{*}$ are only matched with vertices from ICA and ECA on the distal side of the flow divider [see Fig. 4(c)]. In practice, it is convenient to work in the proximal to distal direction so that the ICA and ECA contours are resampled first. The overlapping contours ICA* and $\mathrm{ECA}^{*}$ are then merged to form $\mathrm{CCA}^{*}$, and proximal $\mathrm{CCA}$ contours are resampled so that they contain the same number of vertices as $\mathrm{CCA}^{*}$, with

$$
N_{\mathrm{CCA}}=N_{\mathrm{ICA}}+N_{\mathrm{ECA}}-N_{\text {extra }}
$$

where $N_{\mathrm{CCA}}, N_{\mathrm{ICA}}$, and $N_{\mathrm{ECA}}$ are the number of vertices in the CCA, ICA, and ECA contours, respectively, and $N_{\text {extra }}$ is the number of nonshared vertices.
Once contour vertices have been matched, smoothing splines are fitted along the whole length of the vessel. Because the iterative weighted smoothing spline algorithm outlined earlier is relatively expensive in terms of computation time, and has marginal benefit once centroid-based realignment of the original wall contours has been performed, a uniformly weighted smoothing spline $\left[w_{i}=1\right.$ for all $i$ in (2)] was used instead to provide a faster means of approximating wall surfaces. Fig. 5(b) demonstrates the result of fitting smoothing splines to the vertices of contours segmented from a 3-D ultrasound scan of a patient carotid bifurcation.

\section{QUANTIFICATION OF CAROTID PLAQUE}

\section{A. Extension of Reconstruction Method to Diseased Vessels}

Using the method described above, it is possible to reconstruct the lumen surface in exactly the same way as the vessel wall. When plaque is present, however, it is likely that a higher value of the smoothing parameter, $p_{\text {lumen }}$, is appropriate to preserve irregular features in the plaque surface. In this case, the problem of surface discontinuity arises in disease-free regions, as illustrated in Fig. 6. Example wall and plaque contours, segmented from an original ultrasound slice, are shown in Fig. 6(a). The open plaque contour is defined by a spline, and merging the plaque and wall contours forms the closed lumen contour shown in Fig. 6(b). If the wall and lumen surfaces are then reconstructed independently using different smoothing parameter values, shape changes in disease-free regions of the vessel may lead to geometric discontinuity between the wall and lumen surfaces in these regions. Fig. 6(c) shows the contours generated by reslicing the reconstructed surfaces in the original slice plane. Apart from the obvious lack of correspondence, it is apparent that quantitative plaque measures, such as cross-sectional area and volume, will also be influenced by this effect. For instance, in the example illustrated in Fig. 6, the plaque cross-sectional area would be overestimated.

In order to avoid surface discontinuities, the scheme depicted in Fig. 7 was adopted. First, the vessel wall is reconstructed 


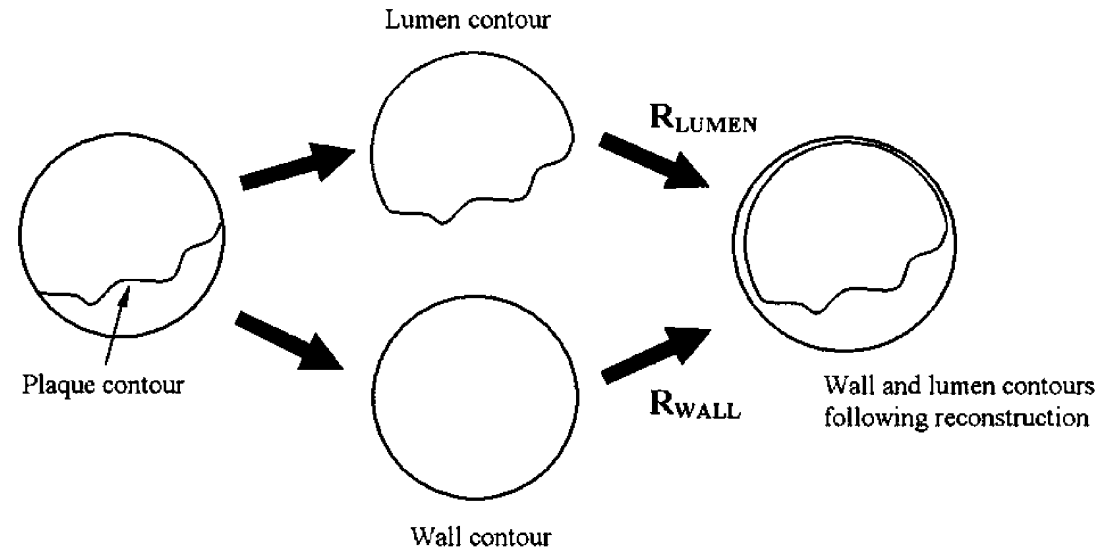

(a)

(b)

(c)

Fig. 6. Inconsistency of vessel wall and lumen surfaces in plaque-free regions following reconstruction. (a) The original segmented wall and plaque contours. (b) A closed lumen contour is formed by merging the plaque contour with the wall contour. (c) Since it can be assumed that the wall is smooth, but no such assumption holds for the lumen (the plaque surface may be irregular), the wall and lumen surfaces are reconstructed independently with different degrees of smoothing. The corresponding reconstruction functions are denoted by $\mathbf{R}_{\text {WALL }}$ and $\mathbf{R}_{\text {LUMEN }}$, respectively. As a consequence, the cross-sectional wall and lumen contours obtained by reslicing the reconstructed surfaces in the original ultrasound slice plane do not coincide in the disease-free portion of the vessel.

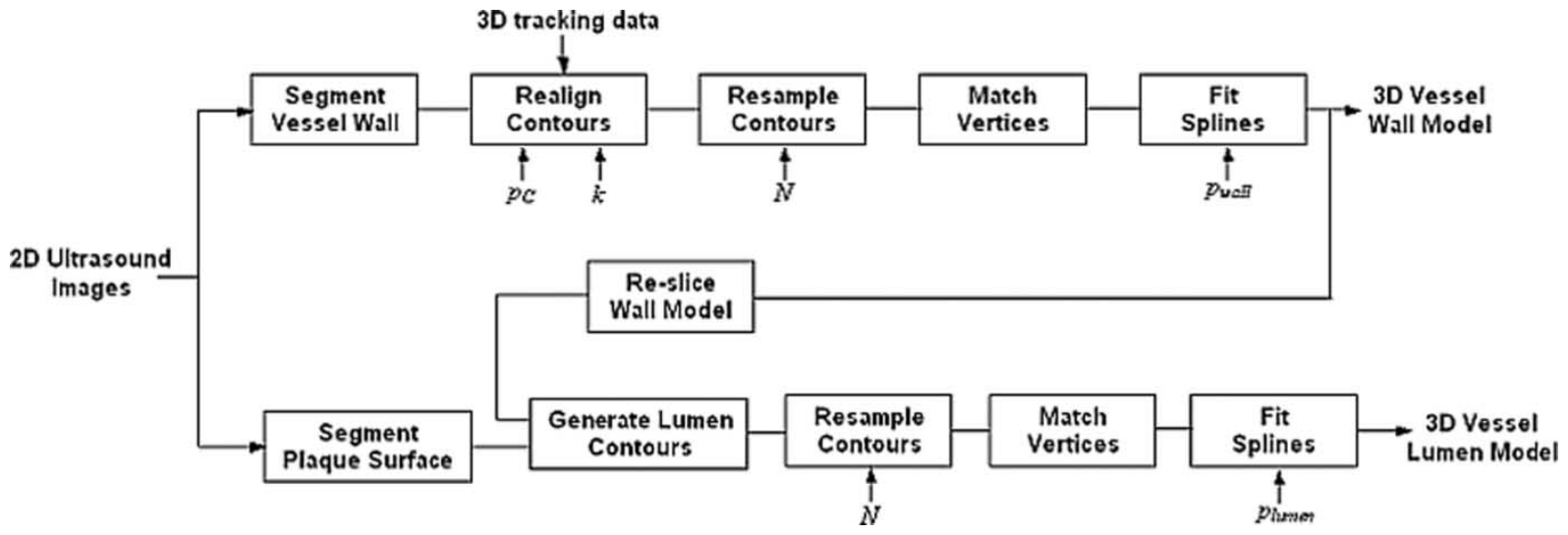

Fig. 7. Block diagram of the coupled wall-lumen reconstruction scheme. $p_{\text {lumen }}$ is the smoothing parameter for the lumen reconstruction.

using an appropriately high degree of smoothing. The reconstructed wall surface is then resliced in planes of the original ultrasound slices and a set of new cross-sectional wall contours are generated (Section IV includes details of the reslicing method used). Now, for each slice, the lumen contour is defined according to the following rules. 1) if no plaque is present, the lumen contour is defined to be identical to the new wall contour. 2) If plaque is present on one side of the vessel wall, for which an open plaque contour (represented by a cubic spline) has been defined, the lumen contour is formed by merging the plaque contour with the new wall contour. 3) If plaque is present all around the circumference of the vessel (i.e., it forms a concentric stenosis), then the original lumen contour defined in the segmentation stage remains unchanged.

\section{B. Determination of the Vessel Central Axes}

The central axis for a segment of vessel may be defined informally as the 3-D curve which passes through the centroids of cross sections along the length of the vessel. This definition was used in the wall contour realignment step described earlier and is adequate for this purpose. However, the geometry of the central axis using this definition is in general dependent on the orientation of the cross sections. Another potential problem occurs around bifurcations, where a change in cross-sectional shape proximal to the bifurcation results in a discontinuity between the parent and daughter vessels.

The first of these problems is addressed by the algorithm illustrated in Fig. 8, which is a 3-D extension of the one described by Smith et al. in [59]. After selecting an initial cut-plane approximately perpendicular to the vessel wall, the algorithm iteratively computes a normalized local direction vector, $\mathbf{v}_{i}$ for the vessel by averaging the unit tangent vectors at spline points intersected by the current cut-plane. After each iteration, the next cut-plane is defined as the plane perpendicular to $\mathbf{v}_{i}$, which passes through the point with position vector $\mathbf{p}_{i+1}$ and is given by

$$
\mathbf{p}_{i+1}=\mathbf{c}_{i}+\beta \mathbf{v}_{i}
$$

where $\mathbf{c}_{i}$ is the position vector of the centroid of the $i$ th contour and $\beta$ is a small constant scalar.

This algorithm has the advantage that the course of a curved vessel is followed, ensuring that the cut-planes are optimally transverse, and was used in this study to compute the central axis curve for sections of the reconstructed carotid arteries with 


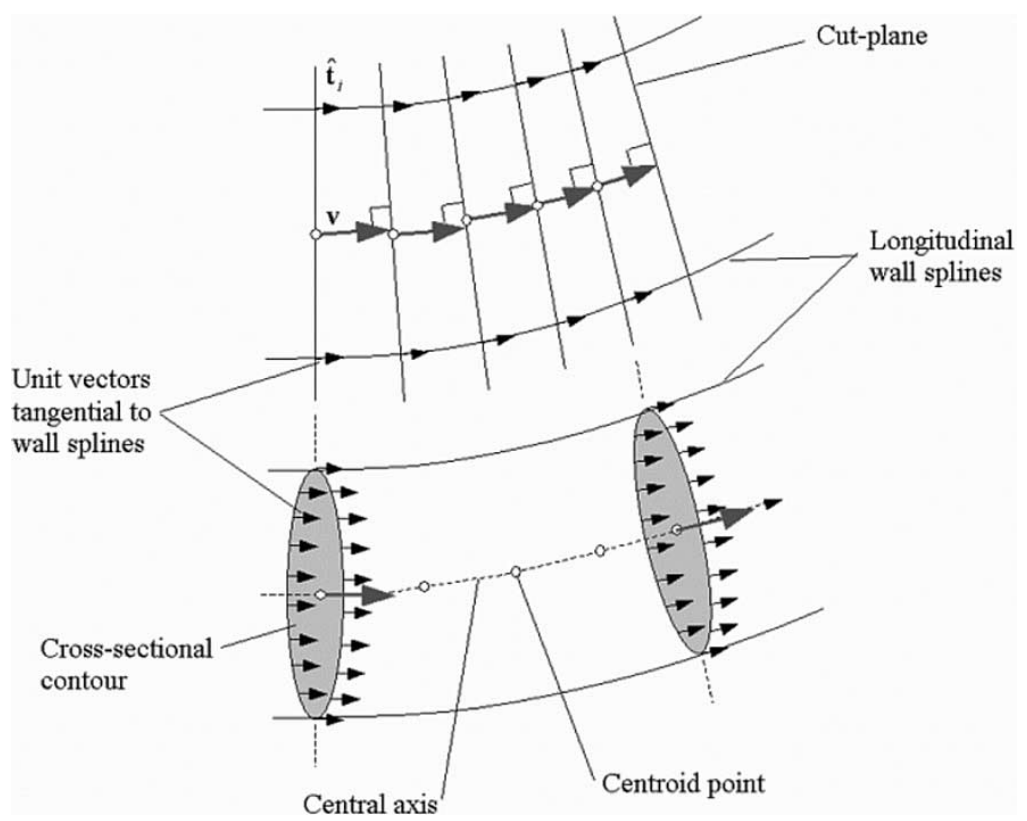

Fig. 8. Determination of the vessel central axis from the reconstructed wall surface. At each iteration of the algorithm a local direction vector, v, (shown as a large arrow) is found by averaging the unit vectors tangential to the longitudinal surface splines at the intersections with the current cut-plane. The cut-plane is then updated by finding the plane that is normal to this vector and passes through a point on the vector a short distance along the vessel.

an approximately circular cross section. In order to minimize the variability in determining whether a wall contour is circular, a dimensionless circularity measure, $F$, defined as

$$
F=\frac{4 \pi A}{C^{2}}
$$

was calculated for each contour, where $A$ and $C$ are the area and circumference of the contour, respectively. It can be seen from (7) that $F=1$ when the contour is circular. A cut-off of $F=0.95$ was used to exclude noncircular cross sections immediately proximal to the flow divider. For the carotid bifurcation, three central axis curves were computed, one for each of the CCA, ICA and ECA segments. Continuity between these curves in the region of the flow divider was achieved by fitting cubic splines between the CCA segment and each of the daughter vessel segments resulting in a single, bifurcating central axis curve.

\section{Planar Reslicing}

Reslicing the reconstructed vessel surfaces in transverse planes is an important operation for quantifying stenosis in 3-D. Using the vessel central axis as a reference, it is possible to reslice the vessel automatically along its length using cut-planes that are perpendicular to the vessel. This, in turn, allows automatic cross-sectional area and/or diameter measurements to be made at any point along the vessel. In principle, automatic analysis in this way is likely to increase the accuracy and precision of finding the maximum degree of diameter/area stenosis.

For the spline-based surface representation adopted here, the reslicing operation is equivalent to finding the set of spline intersections with a transverse cut-plane. The intersections define the vertices of a cross-sectional contour lying within the cut-plane. A cut-plane is uniquely defined by a position vector, $\mathbf{p}_{0}$, and two orthogonal unit vectors, $\hat{\mathbf{v}}_{x}$ and $\hat{\mathbf{v}}_{y}$, which lie within the

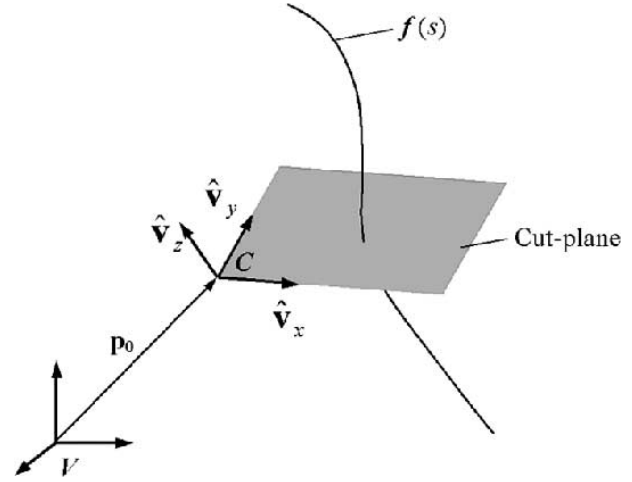

Fig. 9. The intersection of a surface spline, $\boldsymbol{f}(s)$, with a cut-plane. $\boldsymbol{C}$ and $\boldsymbol{V}$ denote 3-D coordinate systems for the cut-plane and reference, repsectively.

plane, as shown in Fig. 9. The position vector, $\mathbf{p}_{V}$, of an arbitrary point in space relative to a reference coordinate system $\boldsymbol{V}$ is then given by

$$
\mathbf{p}_{V}=\mathbf{p}_{0}+a \hat{\mathbf{v}}_{x}+b \hat{\mathbf{v}}_{y}+c \hat{\mathbf{v}}_{z}
$$

where $a, b$, and $c$ are scalars, and

$$
\hat{\mathbf{v}}_{z}=\hat{\mathbf{v}}_{x} \times \hat{\mathbf{v}}_{y} .
$$

Rewriting (8) in homogeneous vector-matrix form yields

$$
\mathbf{p}_{V}={ }^{V} \mathbf{M}_{C} \mathbf{p}_{C}
$$

where $\mathbf{p}_{C}=\left[\begin{array}{llll}a & b & c & 1\end{array}\right]^{\mathrm{T}}$ and

$$
{ }^{V} \mathbf{M}_{C}=\left[\begin{array}{cccc}
\hat{\mathbf{v}}_{x} & \hat{\mathbf{v}}_{y} & \hat{\mathbf{v}}_{z} & \mathbf{p}_{0} \\
0 & 0 & 0 & 1
\end{array}\right]
$$

is the $4 \times 4$ matrix that represents the rigid-body transformation from the within-plane coordinate system, $\boldsymbol{C}$, to $\boldsymbol{V}$. Each piece of the 3-D cubic spline, $\boldsymbol{f}_{V}(s)$, that intersects the cut-plane is 


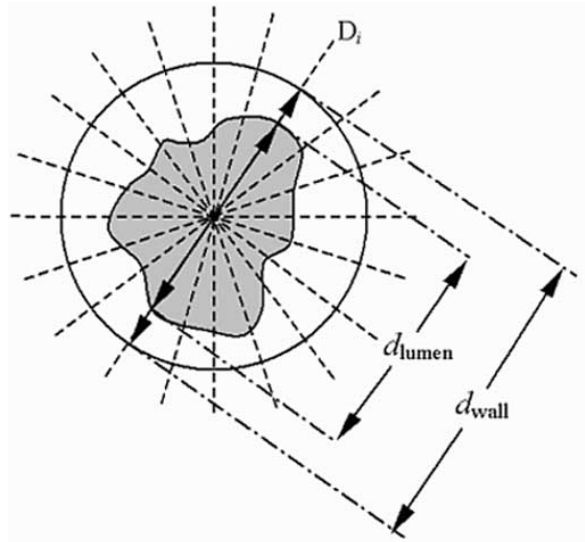

(a)

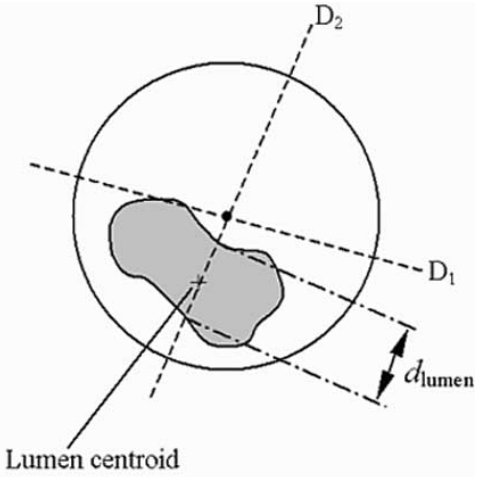

(b)

Fig. 10. Measurement of wall and lumen diameter ( $d_{\text {wall }}$ and $d_{\text {lumen }}$, respectively) in a stenosed artery. (a) When the lumen is concentric, diameters are measured along diametric lines $\left(\mathrm{D}_{i}\right)$ passing through the centroid of the vessel wall. (b) When the lumen is eccentric, however, lumen diameters measured along some diametric lines, e.g., $\mathrm{D}_{1}$, may be arbitrarily small leading to an unrealistically high degree of stenosis. In this case, a stable definition of degree of stenosis is obtained by measuring diameters along the line that passes through the wall and lumen centroids $\left(\mathrm{D}_{2}\right)$.

specified by three cubic polynomials in the (arc-length) parameter $s$, and may be expressed as

$$
\boldsymbol{f}_{V}(s, n)=\mathbf{G}_{n}\left[\begin{array}{llll}
s^{3} & s^{2} & s & 1
\end{array}\right]^{\mathrm{T}}=\mathbf{G}_{n} \mathbf{s}
$$

where $n$ denotes the $n$th piece and $\mathbf{G}_{n}$ is a $4 \times 4$ coefficient matrix. Substituting (12) for $\mathbf{p}_{V}$ in (10) and rearranging gives

$$
\boldsymbol{f}_{C}(s, n)={ }^{V} \mathbf{M}_{C}^{-1} \mathbf{G}_{n} \mathbf{s} .
$$

Now, where the spline and cut-plane intersect, the $z$ component of $\boldsymbol{f}_{C}$ must be zero. Hence, inspection of (13) indicates that finding the point of intersection reduces to finding the values for $s$ and $n$ for which $Q_{n}(s)=0$, where $Q_{n}$ is a cubic polynomial in $s$. The root of $Q_{n}$ can be computed efficiently by first determining the piece that is known to intersect the plane by evaluating $Q_{n}$ at the breaks between pieces. Assuming that a piece intersects the cut-plane just once, the initial condition for intersection then becomes

$$
Q_{n}\left(\xi_{n}\right) Q_{n+1}\left(\xi_{n+1}\right) \leq 0
$$

where $\xi_{n}$ is $n$th break (i.e., the value of $s$ at the beginning of the $n$th piece of a spline).

\section{Plaque Quantification}

A number of quantitative measurements can be determined after reslicing a vessel reconstruction serially along its length. Included in these are cross-sectional area or diameter stenosis, plaque volume and plaque length. When considering degree of stenosis, it is convenient to introduce the following generalized definition of the percentage stenosis index $S_{H}$ :

$$
S_{H}=100 \times\left(1-\frac{H_{\text {lumen }}}{H_{\text {ref }}}\right)
$$

where $H_{\text {lumen }}$ is some measurement of the lumen and $H_{\text {ref }}$ is the corresponding reference measurement. These measurements may be either diameter, area or volume. When scanning the carotid arteries with ultrasound, the field of view is limited in practice due to the presence of the mandible, which often limits the extent of the ICA that can be practically imaged. For this reason, a definition of $S_{H}$ where $H_{\text {ref }}$ is the local cross-sectional diameter or area of the vessel wall within a slice is more appropriate for 3-D ultrasound than, say, the NASCET definition given earlier.

Both cross-sectional wall/lumen area and diameter can be computed rapidly from the spline-based reconstruction. For a concentric stenosis a large number of diameter measurements can be computed automatically over a range of orientations [see Fig. 10(a)]. However, as Fig. 10(b) illustrates, a problem arises when finding the minimum lumen diameter for an eccentric stenosis. When the lumen diameter is measured along a line passing through the centroid of the vessel wall contour, its magnitude decreases to zero as the line is rotated and clips the edge of the lumen contour. Consequently, an algorithm that naively finds the minimum lumen diameter will result in an erroneous degree of stenosis near to $100 \%$ being calculated. To overcome this difficulty, the diameter-based stenosis index was calculated using the diameter measured along a line that passes through the centroids of both the wall and lumen contours when the wall centroid lies outside of the lumen contour, as shown in Fig. 10(b).

Although single-valued measures of stenosis are widely used, they convey no information about the distribution of disease within a vessel. As true transverse measurements can be computed anywhere along a vessel from a 3-D reconstruction, it is a simple matter to obtain a stenosis profile as function of length along the vessel. In this way, quantitative information on plaque distribution is obtained, which may be clinically useful. Furthermore, a volumetric stenosis index may be defined, where $H_{\text {lumen }}$ and $H_{\text {ref }}$ in (15) are the lumen and wall volumes within a section of vessel. Using this definition, $S_{H}$ then represents a gross measure of the "plaque burden," which is also a potentially useful parameter for clinical assessment. In this study, volumes were calculated using a simple planimetric method and cross-sectional contours obtained by reslicing the vessel surface models. 


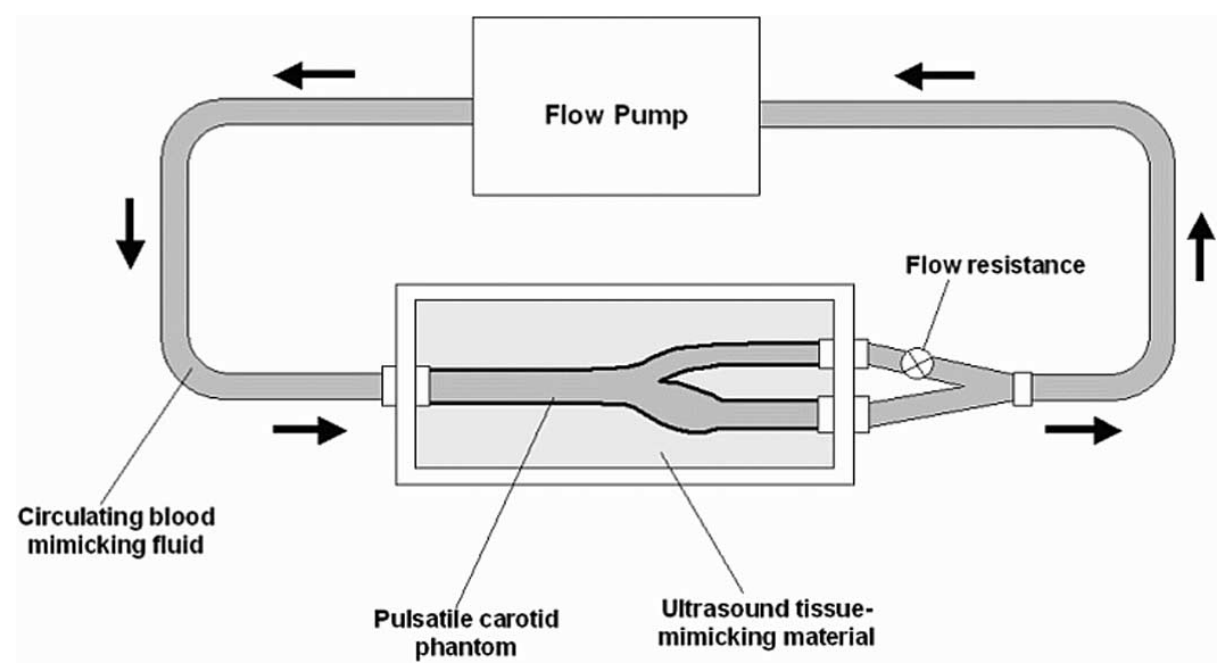

Fig. 11. Experimental setup used to investigate the accuracy of 3-D ultrasound reconstructions using a pulsatile phantom of the carotid bifurcation. The ends of the phantom were connected to silicone tubing and a blood mimicking fluid circulated using a physiological flow pump.

\section{PHANTOM EXPERIMENTS}

Three anthropomorphic phantoms of the human carotid bifurcation (R.G. Shelley Ltd, Ontario, Canada) were used to assess the accuracy of the reconstruction and quantification algorithms of 3-D ultrasound data in vitro. Each phantom was made from polyester tubing molded into the shape of a carotid bifurcation with dimensions derived by averaging angiographic measurements and embedded in a tissue-mimicking material [69], [70]. The process used to fabricate the phantom vessel is based on a novel technique using the low melting point metal, Cerrolow 117 , and is described in [70]. Smith et al. report a root mean square (rms) fabrication accuracy of $0.160 \mathrm{~mm}$ in the phantom lumen radius, estimated by comparing the specified dimensions with those measured from a radiograph at 100 locations [69]. The computer-aided-design (CAD) model used to machine the lumen casts for the phantoms were used as the ground truth geometry against which the accuracy of 3-D ultrasound reconstructions could be compared.

One carotid phantom with a normal geometry and two phantoms with eccentric ICA stenoses of 30 and $70 \%$ (determined using the NASCET method [13]) were used. Each phantom was placed in turn at the head of an examination couch in such a position and orientation to approximate the anatomical location of a patient carotid bifurcation. Fig. 11 illustrates the experimental setup used for imaging the phantoms. Silicone tubing was connected to the ends of the phantom and a blood-mimicking fluid (R.G. Shelley Ltd, Ontario, Canada) pumped through it using a physiological flow pump (Compuflow 1000 Flow System, R.G. Shelley Ltd, Ontario, Canada.) A physiologically realistic pressure waveform was generated by the pump to produce fluid velocities similar to those observed in vivo in the carotid arteries. Each phantom was scanned three times using the 3-D ultrasound system. Image acquisition was gated using a TTL trigger signal generated by the flow pump coincident with the peak of a simulated ECG R-wave. Acquired B-mode images were segmented and reconstructed using a value of 0.75 for the smoothing parameter. Examples of reconstructed phantom bifurcations are shown in Fig. 12.

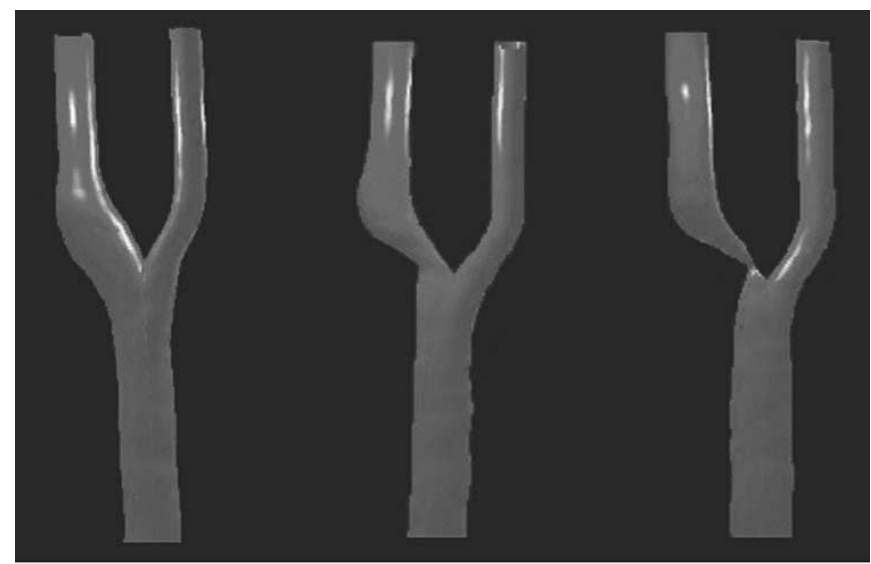

(a)

(b)

(c)

Fig. 12. 3-D ultrasound reconstructions of the carotid artery phantoms used in this study. (a) Normal geometry. (b) Eccentric stenosis $(30 \%)$ in the ICA. (c) Eccentric stenosis $(70 \%)$ in the ICA

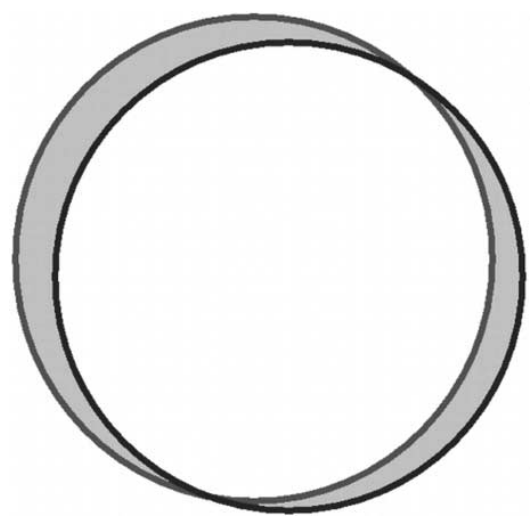

Fig. 13. The area of the region between two overlapping contours (shaded) was calculated to provide a measure of reconstruction accuracy.

For each phantom, a further 3-D reconstruction was obtained from the CAD data and used to estimate the geometric accuracy of each 3-D ultrasound reconstruction. The first step in the geometric analysis was to align each 3-D ultrasound reconstruction 
TABLE I

ERrors in 3-D Ultrasound RECONSTRUCTIONS OF CAROTID BIFURCATION PHANTOMS

\begin{tabular}{lcccc}
\hline \hline \multicolumn{1}{c}{ Phantom } & $\begin{array}{c}\text { RMS registration } \\
\text { error for vessel } \\
\text { central axis points } \\
(\mathrm{mm})\end{array}$ & $\begin{array}{c}\text { Error in cross-sectional area } \\
\left(\mathrm{mm}^{2}\right)\end{array}$ & $\begin{array}{c}\text { Error in non-overlapping } \\
\text { cross-sectional area } \\
\left(\mathrm{mm}^{2}\right)\end{array}$ & $\begin{array}{c}\text { Error in lumen } \\
\text { volume }\left(\mathrm{mm}^{3}\right)\end{array}$ \\
\hline Normal geometry, Scan 1 & 0.24 & $0.32 \pm 1.61(0.62 \pm 4.30 \%)$ & $6.94 \pm 3.23(11.47 \pm 2.50 \%)$ & $19.86(0.83 \%)$ \\
Normal geometry, Scan 2 & 0.18 & $-0.55 \pm 1.40(-1.46 \pm 3.15 \%)$ & $6.02 \pm 2.60(10.13 \pm 2.57 \%)$ & $-32.91(-1.37 \%)$ \\
Normal geometry, Scan 3 & 0.19 & $0.10 \pm 1.39(0.35 \pm 4.31 \%)$ & $6.06 \pm 2.33(10.15 \pm 1.48 \%)$ & $6.79(0.28 \%)$ \\
& & & & \\
$30 \%$ eccentric stenosis, Scan 1 & 0.14 & $-1.16 \pm 1.40(-4.42 \pm 5.57 \%)$ & $4.64 \pm 1.35(10.22 \pm 3.48 \%)$ & $-69.96(-3.60 \%)$ \\
$30 \%$ eccentric stenosis, Scan 2 & 0.17 & $-1.07 \pm 1.48(-3.52 \pm 5.65 \%)$ & $5.04 \pm 1.46(10.99 \pm 3.20 \%)$ & $-64.85(-3.33 \%)$ \\
$30 \%$ eccentric stenosis, Scan 3 & 0.26 & $-1.39 \pm 1.66(-5.09 \pm 5.59 \%)$ & $5.89 \pm 1.82(13.38 \pm 5.25 \%)$ & $-84.48(-4.34 \%)$ \\
& & & & \\
$70 \%$ eccentric stenosis, Scan 1 & 0.39 & $-0.29 \pm 0.92(-1.76 \pm 6.19 \%)$ & $5.38 \pm 1.40(14.72 \pm 6.29 \%)$ & $-17.32(-1.06 \%)$ \\
$70 \%$ eccentric stenosis, Scan 2 & 0.46 & $-1.50 \pm 1.71(-6.46 \pm 9.94 \%)$ & $7.18 \pm 2.83(18.36 \pm 6.39 \%)$ & $-89.98(-5.52 \%)$ \\
$70 \%$ eccentric stenosis, Scan 3 & 0.34 & $-0.01 \pm 1.23(-1.20 \pm 9.78 \%)$ & $5.36 \pm 1.40(14.54 \pm 5.82 \%)$ & $-0.63(-0.04 \%)$
\end{tabular}

Measurements made from a CAD model were used as the ground truth.

All errors are expressed as a mean $\pm \mathrm{SD}$. Percentage errors are given in parentheses.
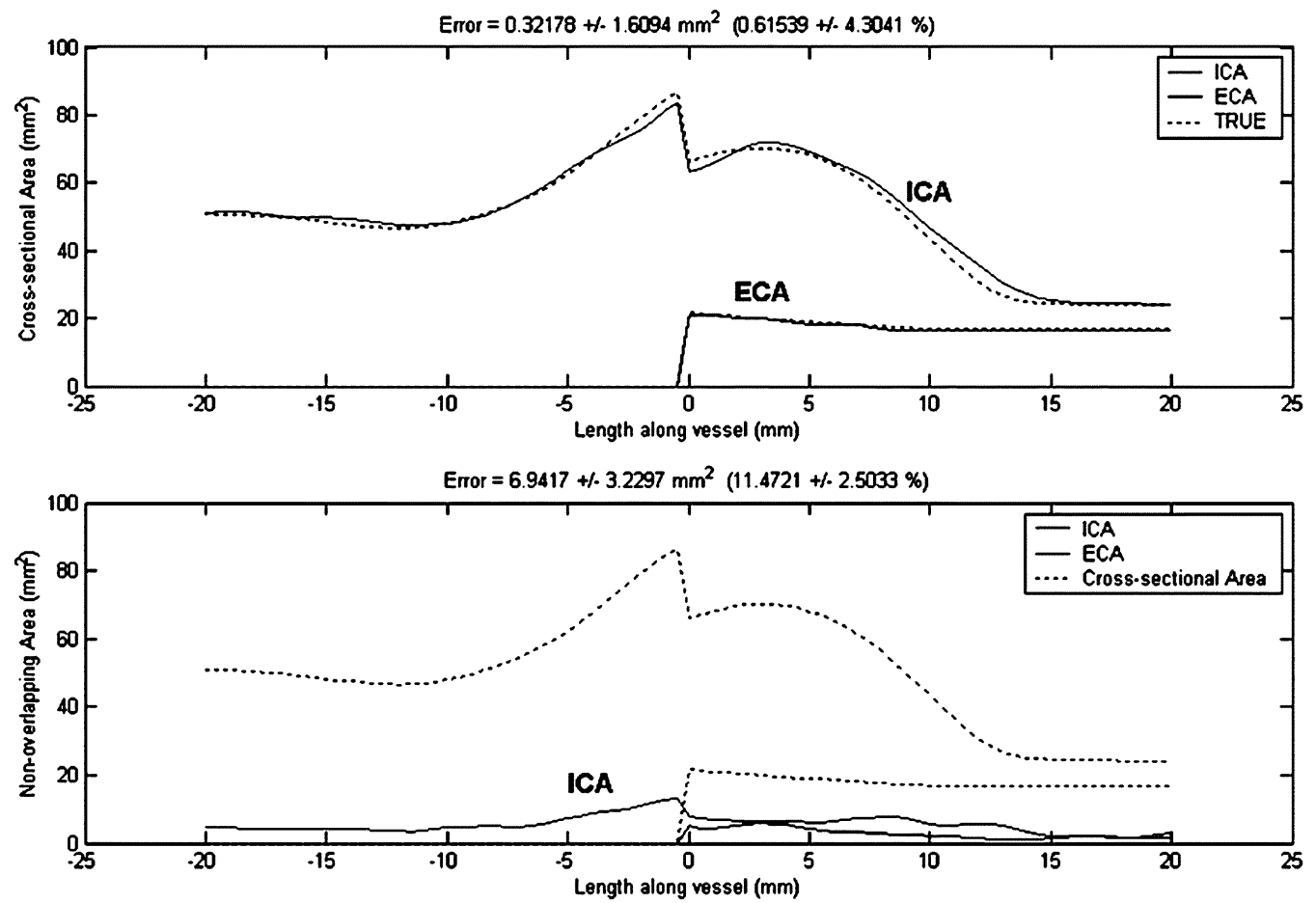

Fig. 14. Sample cross-sectional lumen area plots calculated from a 3-D ultrasound reconstruction of the pulsatile carotid phantom with normal geometry. In each case, errors were calculated with respect to the "true" measurements made from a reconstruction of the CAD data used to fabricate the phantom.

with the corresponding CAD reconstruction. This was achieved by calculating the central axes for each vessel and registering points on the central axes of the 3-D ultrasound model to the corresponding points of the CAD model using a least-squares fit [71]. The rms point-to-point distance following registration of these two point sets was calculated as one measure of geometric similarity between the models.

Once aligned, the CAD and 3-D ultrasound reconstructions were resliced in parallel transverse planes at $0.5-\mathrm{mm}$ intervals to produce a series of cross-sectional contours. The areas of these contours in each slice were computed and compared. The nonoverlapping area, depicted in Fig. 13, between corresponding contours from the $\mathrm{CAD}$ and ultrasound models was also calculated as a further, more stringent, measure of geometric similarity. The numerical errors for all 9 reconstructions are summarized in Table I, and sample area plots for each phantom are shown in Figs. 14-16. It can be seen from the results presented that, overall, the phantom vessel surfaces reconstructed from 3-D ultrasound images were geometrically very similar to the surfaces reconstructed from CAD data.

Three stenosis indexes were also computed for each phantom reconstruction, summarized in Table II. Since the design of phantoms meant that only the vessel lumen could be imaged (although for the purposes of 3-D reconstruction the phantom lumen was treated as a smooth vessel wall), each local stenosis index was calculated by using a corresponding measurement 

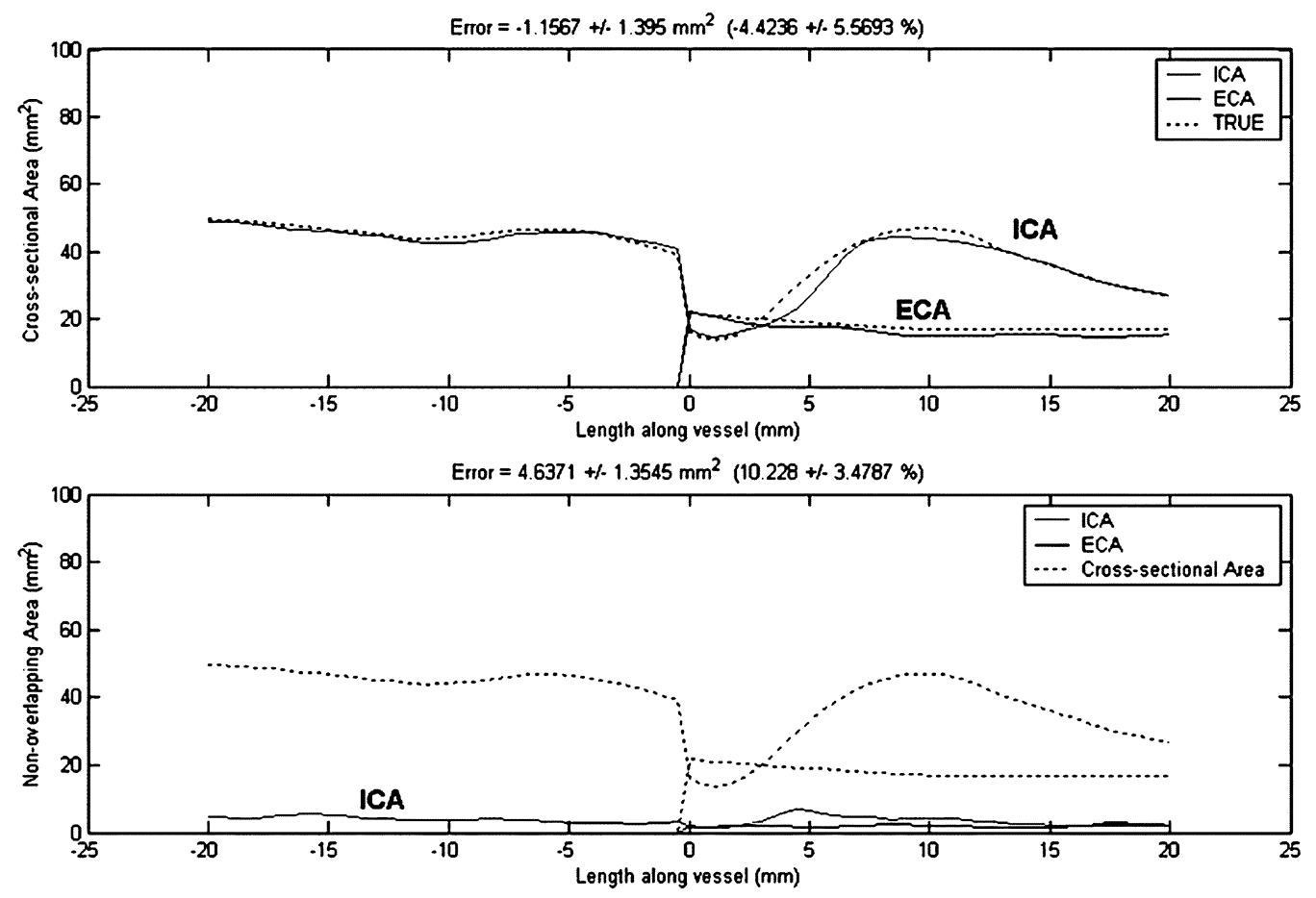

Fig. 15. Sample cross-sectional lumen area plots calculated from a 3-D ultrasound reconstruction of the pulsatile carotid phantom with $30 \%$ eccentric stenosis in the ICA. In each case, errors were calculated with respect to the "true" measurements made from a reconstruction of the CAD data used to fabricate the phantom.
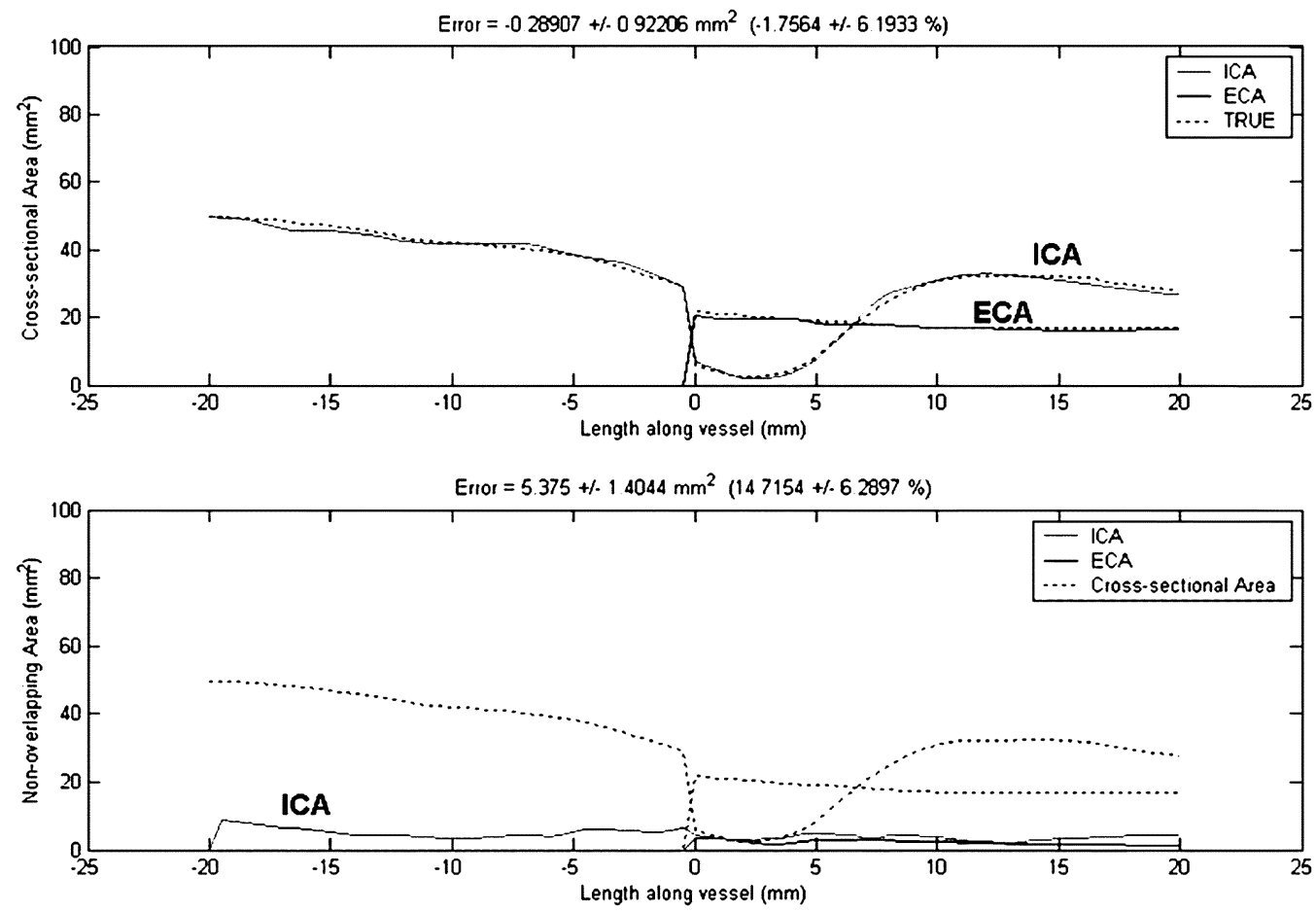

Fig. 16. Sample cross-sectional lumen area plots calculated from a 3-D ultrasound reconstruction of the pulsatile carotid phantom with $70 \%$ eccentric stenosis in the ICA. In each case, errors were calculated with respect to the "true" measurements made from a reconstruction of the CAD data used to fabricate the phantom.

from the CAD reconstruction of the normal carotid bifurcation as the reference parameter. Again, the results presented in Table II indicate a good agreement between the vessel geometry reconstructed from 3-D ultrasound images and the "true" CAD-derived geometry.

\section{Patient Evaluation}

\section{A. Patient Data Sets}

In order to assess the applicability of the techniques described for carotid plaque quantification, eight different carotid bifur- 
TABLE II

Stenosis Indices (\%) CALCULATED From 3-D Ultrasound ReCONSTRUCTIONS OF CAROTID BifurCATION PHANTOMS

\begin{tabular}{|c|c|c|c|}
\hline Phantom & $\begin{array}{l}\text { NASCET diameter } \\
\text { stenosis index }(\%)^{\mathrm{a}}\end{array}$ & $\begin{array}{c}\text { Local diameter stenosis } \\
\text { index }(\%)^{\mathrm{a}}\end{array}$ & $\begin{array}{c}\text { Local area stenosis } \\
\text { index }(\%)^{\mathrm{b}}\end{array}$ \\
\hline $30 \%$ eccentric stenosis, Scan 1 & $25.70(27.87)$ & $53.42(54.78)$ & $78.29(79.55)$ \\
\hline $30 \%$ eccentric stenosis, Scan 2 & $29.37(27.87)$ & $55.72(54.78)$ & $80.28(79.55)$ \\
\hline $30 \%$ eccentric stenosis, Scan 3 & $30.79(27.87)$ & $56.61(54.78)$ & $81.06(79.55)$ \\
\hline $70 \%$ eccentric stenosis, Scan 1 & $73.76(69.45)$ & $83.70(81.02)$ & $97.29(96.40)$ \\
\hline $70 \%$ eccentric stenosis, Scan 2 & $73.73(69.45)$ & $83.68(81.02)$ & $97.30(96.40)$ \\
\hline $70 \%$ eccentric stenosis, Scan 3 & $70.23(69.45)$ & $81.51(81.02)$ & $96.53(96.40)$ \\
\hline
\end{tabular}

Values determined from the CAD reconstruction are given in parentheses.

${ }^{a}$ Calculated using the mean lumen diameter at the location of greatest narrowing in the internal carotid artery

(i.e. smallest cross-sectional area).

${ }^{\mathrm{b}} \mathrm{Calculated}$ using the vessel area at the location of greatest narrowing in the internal carotid artery.

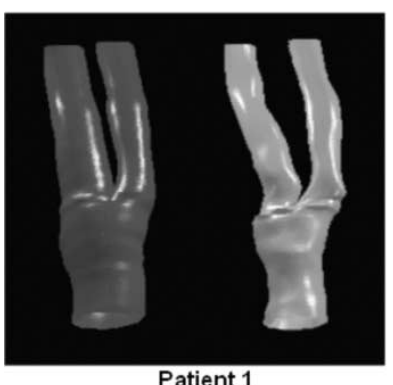

Patient 1

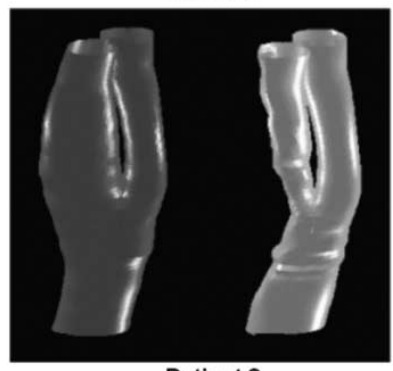

Patient 3

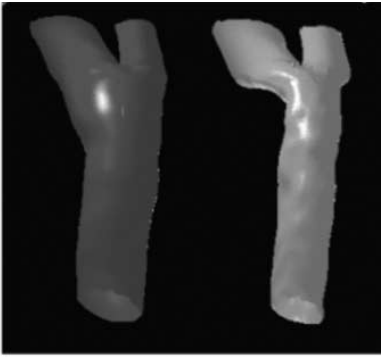

Patient 2

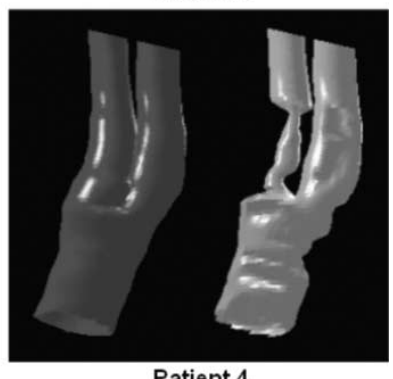

Patient 4

Fig. 17. Surface renderings of the wall and lumen surfaces of four patient carotid bifurcations reconstructed from 3-D ultrasound images. The wall and lumen surfaces are shown on the left and right, respectively, for each patient.

cations from eight patients with known carotid plaque were imaged using 3-D ultrasound. All patients provided written consent to be scanned, and the study was approved by the local hospital Research Ethics Committee. Of the eight datasets obtained, four were deemed to be inadequate for reliable analysis. The images of two of these datasets could not be segmented because of the presence of acoustic shadowing caused by calcified plaque, which precluded visualization of the plaque surface and the intima-media interface over a significant portion of the ICA. Another dataset, obtained from an obese patient, was rejected since the lumen boundary could not be clearly distinguished because of the large depth of the vessel combined with the short length of the ICA that could be imaged. This patient also had marked respiratory problems which led to significant errors in the localization of the vessel due to erratic motion as a result of difficulty with respiration. One further patient suffered from cardiac arrhythmia, which again resulted in unacceptable motion artifacts in the reconstructed vessel.

Fig. 17 shows the reconstructed wall and lumens for the remaining four patients scanned in this study. The plaque cross- sectional area was computed along the complete length of each 3-D reconstruction, plotted in Fig. 18. These data were used to compute both the extent and volume of plaque, and implicitly quantify the distribution of plaque. The local degree of stenosis in the ICA was computed by reslicing the reconstructed ICA surfaces using slices transverse to the vessel central axis. Both diameter and area-based stenosis indexes were calculated, as shown in Fig. 19. A volumetric stenosis index was also calculated for each carotid bifurcation. The numerical results for each patient dataset are summarized in Table III.

\section{DISCUSSION}

From the preliminary in vivo evaluation carried out in this study, it is evident that the carotid arteries of a subgroup of patients are not amenable to assessment using 3-D ultrasound. It is difficult to estimate the true size of this subgroup from such a small sample, but it is likely to comprise arteries which give rise to well-known ultrasound image artifacts, which prevent the vessel wall and lumen from being adequately visualized (in particular, acoustic shadowing or poor signal in the obese patient). Such cases highlight a fundamental limitation of ultrasound imaging, which affects all modes including Doppler ultrasound, rather than a limitation that is peculiar to 3-D ultrasound. Nevertheless, the fact that ultrasound is used very widely for the assessment of carotid plaque, suggests that a significant number of disease arteries can be imaged to the extent that 3-D plaque quantification is feasible in a subgroup of the patient population. This, of course, must be considered within the context of the clear advantages of ultrasound in terms of safety and cost. Since freehand 3-D ultrasound is not a real-time imaging modality, which relies on ECG-gated acquisition to obtain serial images of the vessel at the same phase in the cardiac cycle, patients with cardiac arrhythmia present another group of patients for whom 3-D ultrasound imaging is problematic. Although respiratory motion was not found to introduce significant artifact for the majority of patients scanned in this study, patients who have difficulty in breathing should also be considered for exclusion from 3-D ultrasound assessment to avoid significant motion artifacts being introduced into the reconstruction, unless combined ECG- and respiratory-gated acquisition is employed.

In the work presented here, surface models of diseased carotid arteries were analyzed automatically to provide a number of 

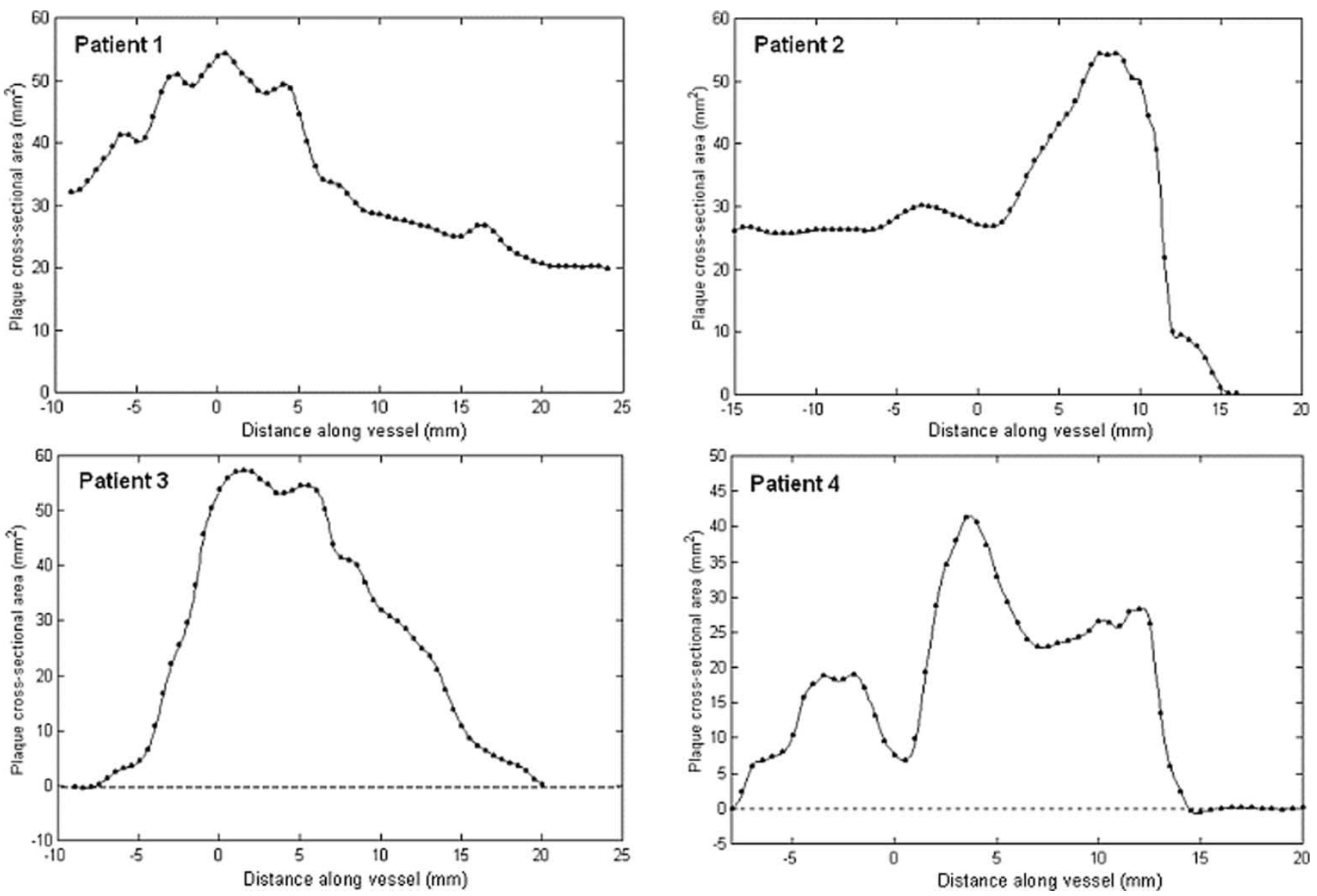

Fig. 18. Cross-sectional carotid plaque area plotted as a function of longitudinal distance along the vessel for each patient.

precise quantitative measures of severity of disease. We have chosen to determine clinically familiar stenosis indexes in addition to new measures that may be readily obtained from the 3-D ultrasound model. Unfortunately, the NASCET method for quantifying degree of stenosis, originally developed for use with planar X-ray angiograms, does not lend itself for use with ultrasound since the length of the proximal ICA that can be imaged is frequently limited to below about $25 \mathrm{~mm}$. Therefore, reliable lumen diameter measurements in the distal ICA are generally impractical. Unlike the local stenosis indexes calculated in this study, the NASCET definition of degree of stenosis is also prone to underestimation of the true severity of disease, as discussed earlier.

The clinical utility of measures, such as plaque volume and volumetric stenosis, remains unknown. It is possible that some of these measures might aid in the identification and management of high-risk patients, either by providing a measure that more accurately reflects the true severity of disease in relation to the dimensions of the vessel wall, or by monitoring plaque progression indicated by changes in serial measurements. 3-D ultrasound analysis also has potential applications for monitoring response to drug therapies. One of the principal advantages of determining the degree of diameter stenosis from a 3-D reconstruction is the ability to precisely select transverse planes perpendicular to the vessel central axis. This has the potential to greatly reduce the variability associated with measurements made during conventional 2-D ultrasound imaging and increase the accuracy, although this has yet to be confirmed from clinical studies beyond the scope of this paper.

Due to the difficulty of reliable automatic or semi-automatic tools for segmenting diseased carotid arteries, in this study, vessel contours were delineated manually on acquired 2-D ultrasound images. Although some measures were taken to speed up the segmentation process, such as using an elliptical approximation for vessel wall cross sections, the time taken to segment wall and lumen contours is currently a significant impediment to the introduction of 3-D ultrasound into clinical practice. For this study, a patient 3-D data set took up to $40 \mathrm{~min}$ to segment, depending on the extent and geometric complexity of plaque. However, it should be borne in mind that for the patient data sets presented in this paper, most of the images acquired during a single sweep were segmented. In practice, this is not strictly necessary, and the 3-D reconstruction could be reasonably confined to the region where plaque is most significant in terms of size or in relation to clinical symptoms. Targeting the reconstruction in this way would reduce the segmentation time considerably, thus making the technique more practical for clinical purposes, but potentially at the expense of a more complete analysis of the total amount of plaque in the carotid arteries. The additional time required to perform a 3-D carotid ultrasound scan is minimal-a few minutes. Therefore, 3-D investigations may be easily added to the end of a routine ultrasound investigation without patient throughput being affected. With the above limitations in mind, 

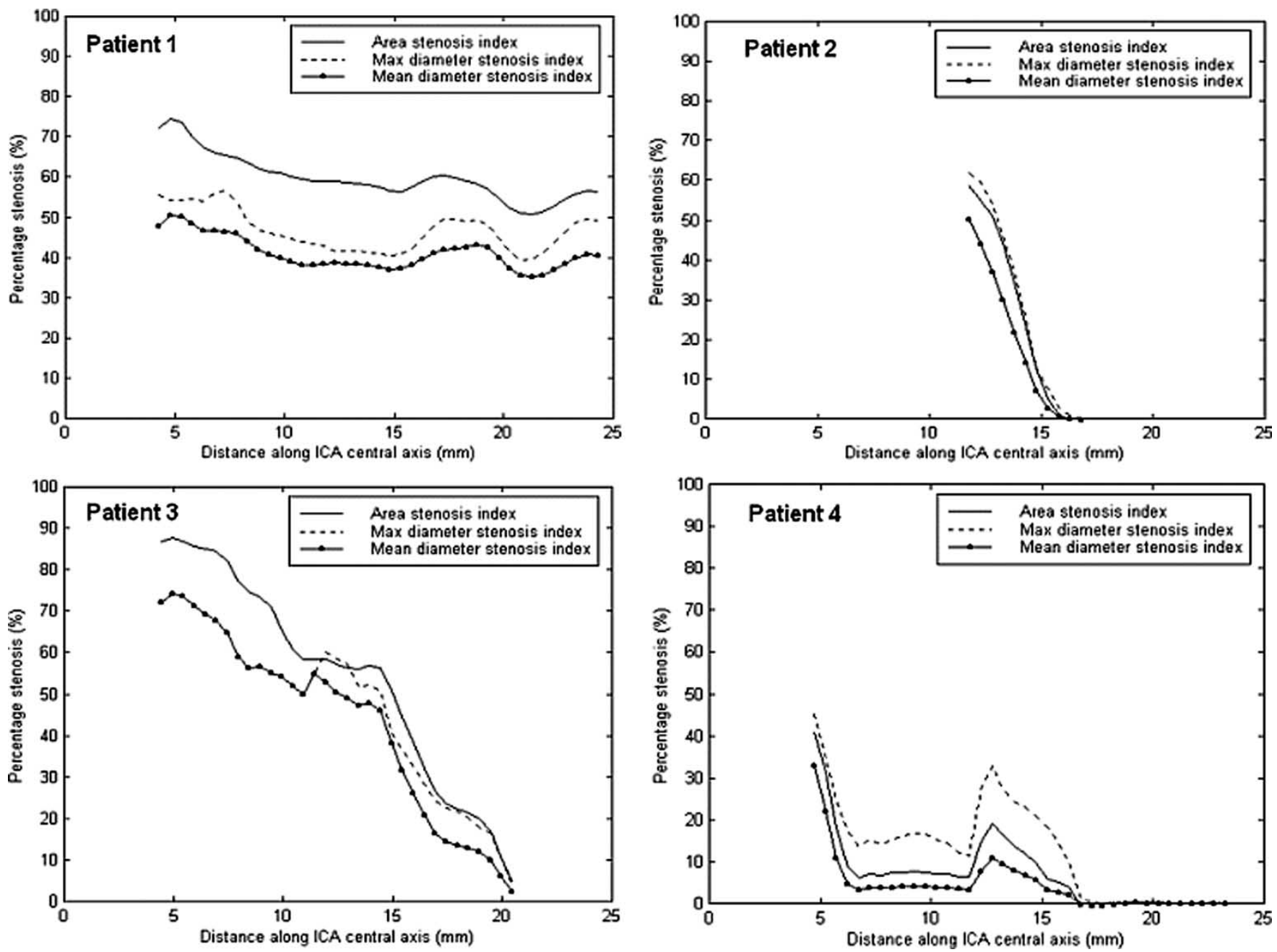

Fig. 19. Degree of stenosis plotted as a function of distance along the central axis of the ICA for each patient. At each point along the vessel, the degree of stenosis was calculated within a slice perpendicular to the central axis using three different definitions, as shown. The area, mean and maximum diameter stenosis indexes were calculated using the lumen cross-sectional area, and mean and minimum lumen diameters for each slice, respectively.

TABLE III

Quantitative Measures of Carotid Plaque Determined From 3-D Ultrasound Reconstructions for Four Patient ARTERIES

\begin{tabular}{|c|c|c|c|c|c|c|}
\hline Patient & $\begin{array}{c}\text { Plaque } \\
\text { extent }(\mathrm{mm})\end{array}$ & $\begin{array}{c}\text { Plaque } \\
\text { volume }\left(\mathrm{mm}^{3}\right)\end{array}$ & $\begin{array}{l}\text { Maximum local degree } \\
\text { of stenosis in the ICA } \\
\text { based on minimum } \\
\text { lumen diameter }(\%)\end{array}$ & $\begin{array}{c}\text { Maximum local degree } \\
\text { of stenosis in the ICA } \\
\text { based on mean lumen } \\
\text { diameter }(\%)\end{array}$ & $\begin{array}{c}\text { Maximum local } \\
\text { degree of stenosis } \\
\text { in the ICA based on } \\
\text { lumen area }(\%)\end{array}$ & $\begin{array}{c}\text { Volumetric } \\
\text { stenosis for } \\
\text { reconstructed } \\
\text { bifurcation }(\%)\end{array}$ \\
\hline 1 & 32.50 & 1120.76 & 57 & 50 & 75 & 56 \\
\hline 2 & 30.40 & 915.94 & 62 & 51 & 59 & 41 \\
\hline 3 & 27.98 & 781.93 & 74 & 74 & 88 & 42 \\
\hline 4 & 22.34 & 445.02 & 45 & 33 & 44 & 33 \\
\hline
\end{tabular}

the technique is currently restricted to use within research studies where a detailed description of 3-D vascular geometry is required. One application where the current time overhead for generating 3-D reconstructions of the carotid bifurcation may be justified, however, is in the surgical planning of carotid endarterectomy.

In our reconstruction method, the geometry of the flow divider is defined implicitly by a number of intersecting surface splines. This approach is similar to that of Yim and colleagues, where bifurcating vessel surfaces were generated by merging overlapping vessel segments [65]. This solution was chosen because it is normally very difficult to locate the position of the flow-divider precisely from acquired ultrasound slices, due partly to the low resolution of ultrasound images in the elevational direction, and partly to the fact that during freehand acquisition images are typically obtained immediately proximal and distal to the flow divider, but not precisely at the flow divider. Hence, interpolation is necessary to reconstruct the surface geometry between these slices. Our solution requires the user to define two overlapping contours for the image immediately proximal to the flow divider, which is a quick and simple addition to the segmentation process. Once the vessel 
TABLE IV

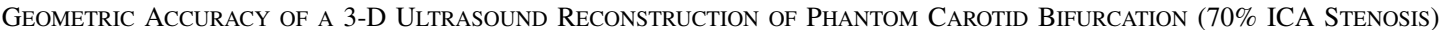
USING A RANGE OF DIFFERENT VALUES FOR THE SMOOTHING PARAMETER

\begin{tabular}{ccccc}
\hline \hline $\begin{array}{c}\text { Smoothing } \\
\text { parameter, } p_{\text {wall }}\end{array}$ & $\begin{array}{c}\text { RMS registration } \\
\text { error for vessel } \\
\text { central axis points } \\
(\mathrm{mm})\end{array}$ & $\begin{array}{c}\text { RMS error in } \\
\text { cross-sectional area } \\
\left(\mathrm{mm}^{2}\right)\end{array}$ & $\begin{array}{c}\text { RMS error in } \\
\text { non-overlapping } \\
\text { rross-sectional area }_{\left(\mathrm{mm}^{2}\right)}\end{array}$ & $\begin{array}{c}\text { Error in lumen } \\
\text { volume }\left(\mathrm{mm}^{3}\right)\end{array}$ \\
\hline 0.005 & 0.46 & 2.32 & 7.54 & -40.47 \\
0.010 & 0.41 & 1.41 & 6.47 & -26.23 \\
0.050 & 0.37 & 1.01 & 5.79 & -8.65 \\
0.100 & 0.37 & 1.02 & 5.78 & -6.41 \\
0.500 & 0.37 & 1.11 & 5.97 & -6.32 \\
1.000 & 0.38 & 1.23 & 6.17 & -6.65 \\
& & & \\
\hline \hline
\end{tabular}

surface has been reconstructed, redundant spline segments are removed automatically when the model is resliced in transverse sections to obtain cross-sectional wall/lumen contours.

In general, smoothing splines provide a compact parametric representation and a convenient way of incorporating a variable degree of smoothing into the reconstruction, governed by setting a global smoothing parameter. However, global smoothing may be undesirable in certain circumstances, for example, when localized artifacts or surface features are present. Therefore, extending the algorithm so that local smoothing could be applied would be desirable to extend its applicability.

Another important issue is the choice of the smoothing parameter used in the wall/lumen surface reconstruction. As stated previously, excessive smoothing should not be applied to the lumen in order to preserve irregular plaque surface features (i.e., $p_{\text {lumen }}=1$ ). The vessel wall, on the other hand, may be reasonably assumed to be smooth, and, therefore, some smoothing is justified ( $p_{\text {wall }}=0.75$ was used in this study). To investigate the effect of the smoothing parameter on reconstruction accuracy, the 3-D ultrasound data from the carotid bifurcation phantom with a $70 \%$ eccentric stenosis in the ICA was reconstructed using varying values for $p_{\text {wall }}$. Table IV illustrates the results. It can be seen that the reconstruction accuracy is robust over a relatively wide range of $p_{\text {wall }}$, although this may reflect the artificially high quality of images obtained from scanning a phantom, perhaps in combination with the reduction in slice misregistration due to initial centroid realignment.

The spline-based vessel surface representation is suitable for grid generation for modeling blood flow using CFD, although this application was not the focus of this study. Further details can be found in [72] and [73]. The reconstruction algorithm may be readily applied to vessels other than the carotid arteries. Currently, the method is unable to handle very tortuous vessels, where the $\mathrm{z}$ component of the central axis is not monotonic. It is also unsuitable for very wide bifurcations angles where images intersect the vessel obliquely, and, hence, no closed, elliptical wall contour can be obtained. Both of these limitations could be remedied by including an initial estimate of vessel central axes to guide the segmentation. This approach has been adopted by other researchers, but requires the availability of a volumetric data set, which is can be resliced in arbitrarily oriented planes. In the case of freehand 3-D ultrasound, the inclusion of an initial volume reconstruction step would then be necessary. Although this adds an extra stage of computation, which is avoided in our current implementation, it would allow intersecting images from a densely sampled dataset to be handled automatically and may suppress speckle artifact through 3-D compounding. Although this may make semi- or fully automatic segmentation of the vessel lumen more feasible, it is unlikely to replace the requirement for manual segmentation of the plaque and vessel wall. Thus, in practice, segmentation from (parallel) 2-D slices would still be necessary. An advantage of the approach described in this paper is that a prior volumetric reconstruction is not required, since segmentation is performed directly on the acquired 2-D ultrasound slices. This has the added potential benefit that image artifacts, which can sometimes be introduced by certain volumetric reconstruction methods, are avoided [74], [75].

Experiments on pulsatile carotid bifurcation phantoms suggest that accurate surface reconstructions can be obtained in vitro using our spline-based method, and several indexes of severity of carotid disease can be measured with high accuracy. The phantom carotid bifurcations used in this study may be considered to be anthropomorphic, but differ from real arteries in a few important respects. In particular, they have a planar geometry, with straight sections, and plaque is modeled by a smoothly reducing lumen cross section without the presence of a wall interface. Although the phantoms were considered adequate to demonstrate the upper bounds on the accuracy of our vessel reconstruction and quantification algorithms, more anatomically realistic phantoms, perhaps constructed from post-mortem specimens, would enable the methods to be evaluated under more realistic conditions. A comparison between the in vivo geometry of the normal carotid bifurcation reconstructed from 3-D ultrasound and black-blood MRA images, using the method described in this paper, can be found in [76].

\section{ACKNOWLEDGMENT}

The authors would like to thank M. Kerle for assistance in finding patient volunteers for this study.

\section{REFERENCES}

[1] P. Picot, D. Rickey, R. Mitchell, R. Rankin, and A. Fenster, "Three-dimensional Doppler imaging," Ultrasound Med. Biol., vol. 19, pp. 95-104, 1993

[2] A. Fenster, D. Lee, S. Sherebrin, R. Rankin, and D. Downey, "Threedimensional ultrasound imaging of the vasculature," Ultrasonics, vol. 36, pp. 629-633, 1998. 
[3] Z. Guo and A. Fenster, "Three-dimensional power Doppler imaging: A phantom study to quantify vessel stenosis," Ultrasound Med. Biol., vol. 22, pp. 1059-1069, 1996.

[4] C. Ritchie, W. Edwards, L. Mack, D. Cyr, and Y. Kim, "Three-dimensional ultrasonic angiography using power-mode Doppler," Ultrasound Med. Biol., vol. 22, pp. 277-286, 1996.

[5] K. Wang, R. Dutton, and C. Taylor, "Improving geometric model construction for blood flow modeling," IEEE Eng. Med. Biol. Mag., vol. 18, pp. 33-39, 1999

[6] U. G. Schulz and P. M. Rothwell, "Major variation in carotid bifurcation anatomy: A possible risk factor for plaque development?," Stroke, vol. 32, pp. 2522-2529, Nov. 2001.

[7] J. Moore, D. Steinman, and C. Ethier, "Computational blood flow modeling: Errors associated with reconstructing finite element models from magnetic resonance images," J. Biomech., vol. 31, pp. 179-184, 1998.

[8] Q. Long, X. Y. Xu, B. Ariff, S. Thom, A. Hughes, and A. Stanton, "Reconstruction of blood flow patterns in a human carotid bifurcation: A combined CFD and MRI study," J. Magn. Reson. Imag., vol. 11, pp. 299-311, 2000.

[9] G. Ailawadi, J. C. Stanley, S. Rajagopalan, and G. R. Upchurch Jr., "Carotid stenosis: Medical and surgical aspects," Clin. Cardiol., vol. 20, pp. 599-609, Nov. 2002.

[10] A. Long, E. Lepoutre, E. Corbillon, and A. Branchereau, "Critical review of non- or minimally invasive methods (Duplex ultrasonography, MR-aniography and CT-angiography) for evaluating stenosis of the proximal internal carotid artery," Eur. J. Vasc. Endovasc. Surg., vol. 24, pp. 43-52, 2002.

[11] P. M. Rothwell, "Incidence, risk factors and prognosis of stroke and TIA: The need for high-quality, large-scale epidemiological studies and metaanalyses," Cerebrovasc. Dis., vol. 16, pp. 2-10, 2003.

[12] K. Modaresi, T. Cox, P. Summers, J. Jarosz, H. Verma, P. Taylor, and T. Padayachee, "Comparison of intra-arterial digital subtraction angiography, magnetic resonance angiography and Duplex ultrasonography for measuring cartotid artery stenosis," Br. J. Surg., vol. 86, pp. 1422-1426, 1999.

[13] North American Symptomatic Carotid Endarterectomy Trial (NASCET) Steering Committee, "North american symptomatic carotid endarterectomy trial: Methods, patient characteristics, and progress," Stroke, vol. 22, pp. 711-720, 1991.

[14] North American Symptomatic Carotid Endarterectomy Trial Collaborators, "Beneficial effect of carotid endarterectomy in symptomatic patients with high-grade carotid stenosis," N. Eng. J. Med., vol. 325, pp. 445-453, 1991.

[15] European Carotid Surgery Trialists' Collaborative Group, "Randomised trial of endarterectomy for recently symptomatic carotid stenosis: Final results of the MRC european carotid surgery trial," Lancet, vol. 351, pp. 1379-1386, 1998.

[16] P. M. Rothwell, S. A. Gutnikov, and C. P. Warlow, "Reanalysis of the final results of the European Carotid Surgery Trial," Stroke, vol. 34, pp. 514-523, Feb. 2003.

[17] P. M. Rothwell, M. Eliasziw, S. A. Gutnikov, A. J. Fox, D. W. Taylor, M. R. Mayberg, C. P. Warlow, and H. J. Barnett, "Analysis of pooled data from the randomised controlled trials of endarterectomy for symptomatic carotid stenosis," Lancet, vol. 361, pp. 107-116, Jan. 2003.

[18] P. Sidhu and P. Allan, "Ultrasound assessment of internal carotid artery stenosis," Clin. Radiol., vol. 52, pp. 654-658, 1997.

[19] W. Zwiebel, "Doppler evaluation of carotid stenosis," in Introduction to Vascular Ultrasonography, Fourth ed, W. Zwiebel, Ed. Philadelphia, PA: Saunders, 1999, pp. 137-154.

[20] A. V. Alexandrov, D. S. Brodie, A. McLean, P. Hamilton, J. Murphy, and P. N. Burns, "Correlation of peak systolic velocity and angiographic measurement of carotid stenosis revisited," Stroke, vol. 28, pp. 339-342, 1997.

[21] M. Hunink, J. Polak, M. Barlan, and D. O'Leary, "Detection and quantification of carotid artery stenosis: Efficacy of various Doppler velocity parameters," Amer. J. Roentgenol., vol. 160, pp. 619-625, 1993.

[22] P. J. Nederkoorn, W. P. Mali, B. C. Eikelboom, O. E. Elgersma, E. Buskens, M. G. Hunink, L. J. Kappelle, P. C. Buijs, A. F. Wust, L. A. van der, and G. Y. van der, "Preoperative diagnosis of carotid artery stenosis: Accuracy of noninvasive testing," Stroke, vol. 33, pp. 2003-2008, Aug. 2002

[23] G. Moneta et al., "Screening for asymptomatic internal carotid artery stenosis: Duplex criteria for discriminating $60 \%$ to $90 \%$ stenosis," $J$. Vasc. Surg., vol. 21, pp. 989-994, 1995.

[24] J. Carpenter, F. Lexa, and J. Davis, "Determination of Duplex Doppler ultrasound criteria appropriate to the north american symptomatic carotid endarterectomy trial," Stroke, vol. 27, pp. 695-699, 1996.
[25] E. G. Grant, A. J. Duerinckx, S. ElSaden, M. L. Melany, G. Hathout, P. Zimmerman, S. N. Cohen, R. Singh, and J. D. Baker, "Doppler sonographic parameters for detection of carotid stenosis: Is there an optimum method for their selection?," Amer. J. Roentgenol., vol. 172, pp. 1123-1129, 1999.

[26] E. Grant, A. Duerinckx, S. E. Saden, M. Melany, G. Hathout, P. Zimmerman, A. Marumoto, S. Cohen, and J. Baker, "Ability to use Duplex ultrasound to quantify internal carotid arterial stenosis: Fact or fiction?," Radiology, vol. 214, pp. 247-252, 2000.

[27] D. J. Dippel, A. deKinkelder, S. M. Bakker, F. vanKooten, H. vanOverhagen, and P. J. Koudstaal, "The diagnostic value of color Duplex ultrasound for symptomatic carotid stenosis in clinical practice," Neuroradiology, vol. 41, pp. 1-8, 1999.

[28] P. M. Rothwell, R. J. Gibson, R. Villagra, R. Sellar, and C. P. Warlow, "The effect of angiographic technique and image quality on the reproducibility of measurement of carotid stenosis and assessment of plaque surface morphology," Clin. Radiol., vol. 53, pp. 439-443, June 1998.

[29] S. Glagov, E. Weisenberg, C. Zarins, R. Stanunavicius, and G. Kolettis, "Compensatory enlargement of human atherosclerotic coronary arteries," N. Eng. J. Med., vol. 316, pp. 1371-1375, 1986.

[30] V. Dzau and G. Gibbons, "Vascular remodeling: Mechanisms and implications," J. Cardiovasc. Pharmacol., vol. 21, 1993.

[31] G. Gibbons and V. Dzau, "The emerging concept of vascular remodeling," N. Eng. J. Med., vol. 330, pp. 1431-1438, 1994.

[32] E. N. Arnett et al., "Coronary artery narrowing in coronary heart disease: Comparison of cine-angiography and necropsy findings," Ann. Intern. Med., vol. 91, pp. 350-356, 1979.

[33] P. Arbeille, M. H. Bouin-Pineau, and S. Herault, "Accuracy of the main Doppler methods for evaluating the degree of carotid (continuous wave, pulsed wave, and color Doppler)," Ultrasound Med. Biol., vol. 25, pp. 65-73, 1999.

[34] R. D. Henderson, D. A. Steinman, M. Eliasziw, and H. J. M. Barnett, "Effect of contralateral carotid artery stenosis on carotid ultrasound velocity measurements," Stroke, vol. 31, pp. 2636-2640, 2000.

[35] P. R. Hoskins, "Accuracy of maximum velocity estimates made using Doppler ultrasound systems," Br. J. Radiol., vol. 69, pp. 172-177, 1996.

[36] J. Meyer, R. Khalil, N. Obuchowski, and L. Baus, "Common carotid artery: Variability of Doppler US velocity measurements," Radiology, vol. 204, pp. 339-341, 1997

[37] R. Mikkonen, J. Kreula, and P. Virkkunen, "Reproducibility of Doppler ultrasound measurements," Acta Radiologica, vol. 37, pp. 545-550, 1996.

[38] C. Ranke, P. Hendrickx, U. Roth, F. Brassel, A. Creutzig, and K. Alexander, "Color and conventional image-directed Doppler ultrasonography: Accuracy and sources of error in quantitative blood flow measurements," J. Clin. Ultrasound, vol. 20, pp. 187-193, 1992.

[39] C. Ranke, A. Creutzig, H. Becker, and H.-J. Trappe, "Standardization of carotid ultrasound. A hemodynamic method to normalize for interindividual and interequipment variability," Stroke, vol. 30, pp. 402-406, 1999.

[40] R. Scissons et al., "Doppler peak systolic velocity measurements: Correction of angle-dependent errors," J. Vasc. Tech., vol. 21, pp. 233-236, 1997.

[41] A. Steinman et al., "Sources of error in maximum velocity estimation using linear phased-array Doppler systems with steady flow," Ultrasound Med. Biol., vol. 27, pp. 655-664, 2001.

[42] H. G. Beebe, S. X. SallesCunha, R. P. Scissons, S. M. Dosick, R. C. Whalen, S. S. Gale, J. P. Pigott, and A. J. Seiwert, "Carotid arterial ultrasound scan imaging: A direct approach to stenosis measurement," $J$. Vasc. Surg., vol. 29, pp. 838-844, 1999.

[43] A. H. Rotstein, R. N. Gibson, and P. M. King, "Direct B-mode NASCETstyle stenosis measurement and Doppler ultrasound as parameters for assessment of internal carotid artery stenosis," Australas. Radiol., vol. 46, pp. 52-56, Mar. 2002.

[44] C. P. Allott, C. D. Barry, R. Pickford, and J. C. Waterton, "Volumetric assessment of carotid artery bifurcation using freehand-acquired, compound 3D ultrasound," Br. J. Radiol., vol. 72, pp. 289-292, 1999.

[45] A. Delcker and C. Tegeler, "Influence of ECG-triggered data acquisition on reliability for carotid plaque volume measurements with a magnetic sensor three-dimensional ultrasound system," Ultrasound Med. Biol., vol. 24, pp. 601-605, 1998.

[46] A. Delcker and H. Diener, "Quantification of atherosclerotic plaques in carotid arteries by three-dimensional ultrasound," Br. J. Radiol., vol. 67, pp. 672-678, 1994.

[47] _ "3D-ultrasound of the carotid arteries," Eur. J. Ultrasound, vol. 1, pp. 337-344, 1994 
[48] A. Delcker and H. Polz, "3D ultrasound of the carotid artery using a sensor as a space orientation," Cerebrovasc. Dis., vol. 6, p. 2, 1996.

[49] B. Griewing, U. Schminke, C. Morgenstern, ML. Walker, and C. Kessler, "Three-dimensional ultrasound angiography (Power mode) for the quantification of carotid artery atherosclerosis," J. Neuroimag., vol. 7, pp. 40-45, 1997.

[50] J. A. Hossack, T. S. Sumanaweera, S. Napel, and J. S. Ha "Quantitative 3-D diagnostic ultrasound imaging using a modified transducer array and an automated image tracking technique," IEEE Trans. Ultrason. Ferroelect. Freq. Control, vol. 49, pp. 1029-1038, Aug. 2002.

[51] A. Landry and A. Fenster, "Theoretical and experimental quantification of carotid plaque volume measurements made by three-dimensional ultrasound using test phantoms," Med. Phys., vol. 29, pp. 2319-2327, Oct. 2002.

[52] S. Meairs, J. Beyer, and M. Hennerici, "Reconstruction and visualization of irregularly sampled three- and four-dimensional ultrasound data for cerebrovascular applications," Ultrasound Med. Biol., vol. 26, pp. 263-272, 2000.

[53] C. Palombo, M. Kozakova, C. Morizzo, F. Andreuccetti, A. Tondini, P. Palchetti, G. Mirra, G. Parenti, and N. G. Pandian, "Ultrafast threedimensional ultrasound-Application to carotid artery imaging," Stroke, vol. 29, pp. 1631-1637, 1998 .

[54] K. Rosenfield et al., "Three-dimensional reconstruction of human carotid arteries from images obtained during noninvasive B-mode ultrasound examination," Amer. J. Cardiol., vol. 30, pp. 379-384, 1992.

[55] U. Schminke, L. Motsch, B. Griewing, M. Gaull, and C. Kessler, "Threedimensional power-mode ultrasound for quantification of the progression of carotid artery atherosclerosis," J. Neurol., vol. 247, pp. 106-111, 2000.

[56] J. Yao, M. van Sambeek, A. Dall'Agata, L. van Dijk, M. Kozakova, P. Koudstaal, and J. R. T. C. Roelandt, "Three-dimensional ultrasound study of carotid arteries before and after endarterectomy: Analysis of stenotic lesions and surgical impact on the vessel," Stroke, vol. 29, pp. 2026-2031, 1998.

[57] J. Gill, H. Ladak, D. Steinman, and A. Fenster, "Accuracy and variability assessment of a semiautomatic technique for segmentation of the carotid arteries from three-dimensional ultrasound images," Med. Phys., vol. 27, pp. 1333-1342, 2000.

[58] G. Kovalski, R. Beyar, R. Shofti, and H. Azhari, "Three-dimensional automatic quantitative analysis of intravascular ultrasound images," $\mathrm{Ul}$ trasound Med. Biol., vol. 26, pp. 527-537, 2000.

[59] D. F. Leotta, M. Paun, K. W. Beach, T. Kohler, R. Zierler, and D. Strandness Jr., "Measurement of abdominal aortic aneurysms with three-dimensional ultrasound imaging: Preliminary report," J. Vasc. Surg., vol. 33, pp. 700-707, 2001.

[60] D. F. Leotta, J. F. Primozich, K. W. Beach, R. O. Bergelin, R. E. Zierler and D. E. Strandness Jr., "Remodeling in peripheral vein graft revisions: Serial study with three-dimensional ultrasound imaging," J. Vasc. Surg., vol. 37, pp. 798-807, Apr. 2003.
[61] D. F. Leotta, J. Primozich, K. W. Beach, R. Berglein, and D. Strandness Jr., "Serial measurement of cross-sectional area in peripheral vein grafts using three-dimensional ultrasound," Ultrasound Med. Biol., vol. 27, pp. 61-68, 2001.

[62] D. C. Barratt, A. H. Davies, A. D. Hughes, S. A. Thom, and K. N. Humphries, "Optimization and evaluation of an electromagnetic tracking device for high accuracy three-dimensional imaging of the carotid arteries," Ultrasound Med. Biol., vol. 27, pp. 957-968, 2001.

[63] D. Barratt, A. Davies, A. Hughes, S. Thom, and K. Humphries, "Accuracy of an electromagnetic three-dimensional ultrasound system for carotid artery imaging," Ultrasound Med. Biol., 2001.

[64] A. Frangi, W. Niessen, R. Hoogeveen, T. van Walsum, and M. Viergever, "Model-based quantitation of 3-D magnetic resonance angiographic images," IEEE Trans. Med. Imag., vol. 18, pp. 946-956, 1999.

[65] P. Yim, J. Cebral, R. Mullick, H. Marcos, and P. Choyke, "Vessel surface reconstruction with a tubular deformable model," IEEE Trans. Med. Imag., vol. 20, pp. 1411-1421, 2001.

[66] T. O'Donnell, A. Gupta, and T. E. Boult, "A new model for the recovery of cylindrical structures from medical image data," in Proc. Joint Conf. Comp. Vision, Vir. Reality and Robotics in Medicine and Robotics and Comp.-Assisted Surgery, 1997, pp. 223-232.

[67] K. Bühler, P. Felkel, and A. La Cruz, "Geometric Methods for Vessel Visualization and Quantification-A Survey," VRVis Research Center, Vienna, Austria, 035, 2002.

[68] C. de Boor, A Practical Guide to Splines. New York: Springer-Verlag, 1978.

[69] R. Smith, B. Rutt, A. Fox, and R. Rankin, "Geometric characterization of stenosed human carotid arteries," Acad. Radiol., vol. 3, pp. 898-911, 1996.

[70] R. Smith, B. Rutt, and D. Holdsworth, "Anthropomorphic carotid bifurcation phantom for MRI applications," J. Magn. Reson. Imag., vol. 10, pp. 533-544, 2001.

[71] K. S. Arun, T. S. Huang, and S. D. Blostein, "Least-squares fitting of two 3-D point sets," IEEE Trans. Pattern Anal. Machine Intell., vol. PAMI-9, pp. 698-700, May 1987.

[72] A. D. Augst, D. C. Barratt, A. D. Hughes, F. P. Glor, S. A. Thom, and $\mathrm{X}$. Y. Xu, "Various issues relating to computational fluid dynamics simulations of carotid bifurcation flow based on models reconstructed from three-dimensional ultrasound images," Proc. Inst. Mech. Eng. [H], vol 217, pp. 393-403, 2003.

[73] - "Accuracy and reproducibility of CFD-predicted wall shear stress using 3D ultrasound images," Trans. ASME J. Biomech. Eng., vol. 125 , pp. 218-222, 2003.

[74] D. Pretorius, "Artifacts generated by 3D ultrasound data: Clinical applications," Ultrasound Med. Biol., vol. 26, p. A51, 2000.

[75] R. N. Rohling, A. H. Gee, and L. Berman, "A comparison of freehand three-dimensional ultrasound reconstruction techniques," Med. Image Anal., vol. 3, pp. 339-359, 1999.

[76] F. P. Glor, B. Ariff, L. A. Crowe, P. L. Cheong, D. C. Barratt, D. N. Firmin, A. D. Hughes, and S. A. Thom, "Carotid geometry reconstruction: A comparison between MRI and ultrasound," Med. Phys., vol. 30 pp. 3251-3261, 2003. 